\title{
FREQUENCY-LIMITED BALANCED TRUNCATION WITH LOW-RANK APPROXIMATIONS*
}

\author{
PETER BENNER ${ }^{\dagger}$, PATRICK KÜRSCHNER ${ }^{\ddagger}$, AND JENS SAAK ${ }^{\dagger}$
}

\begin{abstract}
In this article we investigate model order reduction of large-scale systems using frequency-limited balanced truncation, which restricts the well known balanced truncation framework to prescribed frequency regions. The main emphasis is put on the efficient numerical realization of this model reduction approach. We discuss numerical methods to take care of the involved matrix-valued functions. The occurring large-scale Lyapunov equations are solved for low-rank approximations for which we also establish results regarding the eigenvalues of their solutions. These results, and also numerical experiments, will show that the eigenvalues of the Lyapunov solutions in frequency-limited balanced truncation often decay faster than those in standard balanced truncation. Moreover, we show in further numerical examples that frequency-limited balanced truncation generates reduced order models which are significantly more accurate in the considered frequency region.
\end{abstract}

Key words. Lyapunov equations, alternating direction implicit, controllability, observability, balanced truncation, model order reduction, rational Krylov subspaces, matrix functions, matrix logarithm

AMS subject classifications. 15A16, 15A18, 15A24, 65F60, 93A15, 93C

DOI. $10.1137 / 15 \mathrm{M} 1030911$

\section{Introduction.}

1.1. The infinite Gramians and balanced truncation. Our investigations are centered around continuous-time, linear, time-invariant (LTI) systems of the form

$$
\begin{aligned}
\dot{x}(t) & =A x(t)+B u(t), \\
y(t) & =C x(t)
\end{aligned}
$$

with $A \in \mathbb{R}^{n \times n}, B \in \mathbb{R}^{n \times m}$, and $C \in \mathbb{R}^{p \times n}$. We assume that $A$ is Hurwitz, i.e., $\Lambda(A) \subset \mathbb{C}_{-}$. Then, the continuous-time, algebraic Lyapunov equations (CALEs)

$$
A P+P A^{T}=-B B^{T}, \quad A^{T} Q+Q A=-C^{T} C
$$

have unique, symmetric, positive semidefinite solutions $P, Q$. Commonly, $P, Q$ are referred to as infinite controllability and observability Gramian of (1.1). Strict positive definiteness of $P$ and $Q$ is obtained if (1.1) is controllable and, respectively, observable. The eigenvalues of the product $P Q$ are system invariants (w.r.t. state space transformations) and are called Hankel singular values of (1.1). Their magnitude constitutes a joint measurement for controllability as well as observability of the corresponding state components and the aim of balanced truncation (BT) [41] is to identify and truncate components that are weakly controllable and observable. This is achieved by transforming (1.1) into a balanced realization, such that $P=Q=\Sigma=\operatorname{diag}\left(\sigma_{1}, \ldots, \sigma_{n}\right)$.

*Submitted to the journal's Methods and Algorithms for Scientific Computing section July 22, 2015; accepted for publication December 14, 2015; published electronically February 18, 2016.

http://www.siam.org/journals/sisc/38-1/M103091.html

${ }^{\dagger}$ Max Planck Institute for Dynamics of Complex Technical Systems, Sandtorstraße 1, 39106 Magdeburg, Germany (benner@mpi-magdeburg.mpg.de, saak@mpi-magdeburg.mpg.de).

${ }^{\ddagger}$ Corresponding author. Max Planck Institute for Dynamics of Complex Technical Systems, Sandtorstraße 1, 39106 Magdeburg, Germany (kuerschner@mpi-magdeburg.mpg.de).

A471 
ALGORITHM 1.1. SQUARE-ROOT BALANCED TRUNCATION (LOW-RANK VERSION).

Input : System matrices $A, B, C$ defining an asymptotically stable dynamical system (1.1).

Output: Matrices $\tilde{A}, \tilde{B}, \tilde{C}$ of the reduced system.

1 Compute low-rank solution factors $Z_{P}, Z_{Q}$ of the solutions of (1.2), such that $P \approx Z_{P} Z_{P}^{T}, Q \approx Z_{Q} Z_{Q}^{T}$.

2 Compute and partition a (thin) singular value decomposition

$$
X \Sigma Y^{T}=\left[\begin{array}{ll}
X_{1} & X_{2}
\end{array}\right] \operatorname{diag}\left(\Sigma_{1}, \Sigma_{2}\right)\left[\begin{array}{ll}
Y_{1} & Y_{2}
\end{array}\right]^{T}=Z_{Q}^{T} Z_{P},
$$

where $\Sigma_{1}=\operatorname{diag}\left(\sigma_{1}, \ldots, \sigma_{r}\right)$ contains the largest $r$ (approximate) Hankel singular values.

3 Construct $T:=Z_{P} Y_{1} \Sigma_{1}^{-\frac{1}{2}}$ and $S:=Z_{Q} X_{1} \Sigma_{1}^{-\frac{1}{2}}$.

4 Generate reduced order model

$$
\tilde{A}:=S^{T} A T, \quad \tilde{B}:=S^{T} B, \quad \tilde{C}:=C T .
$$

Neglecting all states in the balanced realization corresponding to small values $\sigma_{j}$ gives the reduced order model. Obtaining the Gramians is the computationally most demanding part of BT. Solving (1.2) by methods employing dense numerical linear algebra typically leads to a cubic complexity and quadratic memory demands, which is hardly applicable for large-scale systems. If $p, m \ll n$, which we assume in the remainder, it can be shown that the Gramians often have a small numerical rank $[1,22,45,55,3]$. Hence, there exists, e.g., for $P$, low-rank approximations $Z_{P} Z_{P}^{T} \approx P$ with low-rank solution factors $Z_{P} \in \mathbb{R}^{n \times k_{P}}, \operatorname{rank}\left(Z_{P}\right)=k_{P} \ll n$. Several algorithms exist for computing low-rank solution factors of large-scale CALEs; see, for instance, the survey articles $[9,53]$. Having computed $Z_{P}$, and similarly $Z_{Q}$ such that $Z_{Q} Z_{Q}^{T} \approx Q$, BT for (1.1) using low-rank solution factors of the Gramians (1.2) can be sketched as in Algorithm 1.1. It can be shown that the reduced system generated in (1.3) is asymptotically stable ( $\tilde{A}$ is Hurwitz) and that the following error bound holds:

$$
\|H-\tilde{H}\|_{\mathcal{H}_{\infty}}=\max _{\omega \in \mathbb{R}}\left(\|H(\imath \omega)-\tilde{H}(\imath \omega)\|_{2}\right) \leq 2 \sum_{j=r+1}^{n} \sigma_{j},
$$

where

$$
H(s)=C\left(s I_{n}-A\right)^{-1} B, \quad \tilde{H}(s)=\tilde{C}\left(s I_{r}-\tilde{A}\right)^{-1} \tilde{B}
$$

are the transfer function matrices of the original and reduced order systems. It is important to note that both the stability preservation as well as the error bound (1.4) are intrinsically only proven for the case when $Z_{P}, Z_{Q}$ are exact solution factors, i.e., $Z_{P} Z_{P}^{T}=P, Z_{Q} Z_{Q}^{T}=Q$ solve (1.2) exactly. The effects of using inexact Gramians in BT have been investigated, e.g., in $[2,26,58]$, but to the authors' knowledge, precise ramifications of this inexactness are still not well understood.

1.2. Goals and overview of this article. By looking at the error bound (1.4), the above BT framework generates reduced order models that are accurate for all values $\omega \in \mathbb{R}$, which, from an application oriented view, are typically considered as 
frequencies. In several applications, however, the underlying physical or technical system operates only in a small frequency interval $\left[\omega_{1}, \omega_{2}\right]$ of interest. Restricting the BT procedure to this frequency interval has lead to frequency-limited balanced truncation (FLBT), which was proposed in [19]. One motivation for FLBT is that, compared to ordinary BT, by restricting to a small interval $\left[\omega_{1}, \omega_{2}\right]$, we hope to obtain higher accuracies with reduced order models of the same dimension, or to achieve a comparable accuracy with smaller reduced order models inside the interval, while allowing for larger errors outside.

The main purpose of this article is to provide a numerically efficient framework for carrying out FLBT for high-dimensional systems. We start by reviewing the concept of frequency-limited Gramians and show how to formulate a procedure similar to the square root approach in Algorithm 1.1. In section 3, we investigate the eigenvalue decay of the frequency-limited Gramians. For the occurring CALEs, we will, as in the unlimited case, employ low-rank approximations of the solutions. It turns out that FLBT involves, in addition to solving these CALEs, evaluating a nonlinear matrixvalued function. Hence, we discuss in section 4 the efficient numerical treatment of the involved matrix-valued function. It will turn out that it is possible to deal efficiently with both the matrix-valued function and the CALEs in a single algorithm using extended or rational Krylov subspaces with appropriate shifts. Extensions to generalized LTI systems and some comments on further modifications and variations of BT are given in section 5. Numerical experiments in section 6 illustrate the performance of our approaches with respect to the accuracy of the constructed reduced systems and the computational efficiency regarding the generalized CALE (GCALE) solution.

1.3. Notation. In this paper $\mathbb{R}$ and $\mathbb{C}$ denote the real and complex numbers, and $\mathbb{R}_{-}\left(\mathbb{R}_{+}\right), \mathbb{C}_{-}\left(\mathbb{C}_{+}\right)$refer to the set of strictly negative (positive) real numbers and the open left (right) half plane. Likewise, $\mathbb{R}^{n \times m}, \mathbb{C}^{n \times m}$ denote $n \times m$ real and complex matrices. For a complex quantity $X=\operatorname{Re}(X)+\imath \operatorname{Im}(X), \operatorname{Re}(X), \operatorname{Im}(X)$ are its real and imaginary parts, $\imath$ denotes the imaginary unit, and $\bar{X}=\operatorname{Re}(X)-\imath \operatorname{Im}(X)$ is the complex conjugate of $X$. The absolute value of a complex scalar $z \in \mathbb{C}$ is denoted by $|z|$, and $\arg z$ is its argument. By $\alpha_{j}^{\downarrow}$ we refer to the $j$ th largest element in magnitude of a complex set $\left\{\alpha_{i}\right\} \subset \mathbb{C}, i \geq 1$, i.e., the $\alpha_{i}$ 's are assumed to be ordered like $\left|\alpha_{1}\right| \geq \cdots \geq\left|\alpha_{n}\right|$. If not stated otherwise, $\|\cdot\|$ is the Euclidean vector or subordinate matrix norm (spectral norm). Moreover, $A^{T}$ and $A^{H}=\bar{A}^{T}$ are the transpose and complex conjugate transpose of a real and, respectively, complex matrix. The inverse of a nonsingular matrix $A$ is denoted by $A^{-1}$, and $A^{-H}=\left(A^{H}\right)^{-1}$. The identity matrix of dimension $n$ is indicated by $I_{n}$. Symmetric positive (negative) definiteness of symmetric and Hermitian matrices is denoted by $A \succ 0(\prec 0)$. The spectrum of a matrix pair $(A, E)$ is given by $\Lambda(A, E):=\{z \in \mathbb{C}: \operatorname{det}(A-z E)=0\}$, where det is the determinant. The second argument is neglected if $E=I$. The spectral radius of a matrix $A$ is given by $\rho(A):=\max \{|\lambda|, \lambda \in \Lambda(A)\}$.

\section{Balanced truncation in limited frequency intervals.}

2.1. Frequency-limited Gramians. By employing the Fourier transformation, the Gramians (1.2) can be represented in the frequency domain as

$$
P=\frac{1}{2 \pi} \int_{-\infty}^{\infty} \Psi(\imath \nu) B B^{T} \Psi(\imath \nu)^{H} \mathrm{~d} \nu, \quad Q=\frac{1}{2 \pi} \int_{-\infty}^{\infty} \Psi(\imath \nu)^{H} C^{T} C \Psi(\imath \nu) \mathrm{d} \nu
$$

with the resolvent $\Psi(\imath \nu):=(\imath \nu I-A)^{-1}$. Restricting the integration limits in the integrals (2.1) to certain (unions of) intervals $\Omega \subseteq \mathbb{R}$ gives the frequency-limited Gramians $P_{\Omega}, Q_{\Omega}$. 
Definition 2.1 (frequency-limited Gramians [19]). For the system (1.1), the frequency-limited reachability and observability Gramians w.r.t. $\Omega \subset \mathbb{R}$ are defined by

$$
P_{\Omega}=\frac{1}{2 \pi} \int_{\Omega} \Psi(\imath \nu) B B^{T} \Psi(\imath \nu)^{H} \mathrm{~d} \nu, \quad Q_{\Omega}=\frac{1}{2 \pi} \int_{\Omega} \Psi(\imath \nu)^{H} C^{T} C \Psi(\imath \nu) \mathrm{d} \nu .
$$

Since the system (1.1) is defined by real matrices, the considered frequency region should be symmetric w.r.t. zero: $\Omega=-\Omega$, for instance, in the form

$$
\Omega:=\left[-\omega_{2},-\omega_{1}\right] \cup\left[\omega_{1}, \omega_{2}\right], \quad 0 \leq \omega_{1}<\omega_{2}<\infty .
$$

The following Theorems 2.2-2.3 give important representations of $P_{\Omega}$ and $Q_{\Omega}$. Their proofs can be found, e.g., in [19, section 4] and [46, section 3.1].

Theorem 2.2 (CALEs for the frequency-limited Gramians $[19,46]$ ). Consider the system (1.1) and a frequency region $\Omega \subset \mathbb{R}, \Omega=-\Omega$. Then the frequency-limited Gramians $P_{\Omega}$ and $Q_{\Omega}$ are given in the following equivalent ways:

1. Using the ordinary reachability and observability Gramians $P$ and $Q$ from (1.2), it holds that

$$
P_{\Omega}=F_{\Omega} P+P F_{\Omega}^{T}, \quad Q_{\Omega}=F_{\Omega}^{T} Q+Q F_{\Omega}
$$

with

$$
F_{\Omega}:=\frac{1}{2 \pi} \int_{\Omega} \Psi(\imath \nu) \mathrm{d} \nu .
$$

2. One can also express $P_{\Omega}, Q_{\Omega}$ as the solutions of the frequency-limited reachability and observability CALEs

$$
\begin{aligned}
& A P_{\Omega}+P_{\Omega} A^{T}+F_{\Omega} B B^{T}+B B^{T} F_{\Omega}^{T}=0, \\
& A^{T} Q_{\Omega}+Q_{\Omega} A+F_{\Omega}^{T} C^{T} C+C^{T} C F_{\Omega}=0 .
\end{aligned}
$$

The eigenvalues of the product $P_{\Omega} Q_{\Omega}$ are, in analogy to the case $\Omega=\mathbb{R}$, called frequency-limited Hankel singular values. The matrix $F_{\Omega}$ can also be represented via the matrix-valued natural logarithm, which is established in the next theorem. The theorem also shows that $F_{\Omega}$ is a real matrix.

TheOrem 2.3 (expression of $F_{\Omega}[19,46]$ ). The matrix-valued integral (2.5) can be written for $\Omega$ as in (2.3) as

$$
\begin{aligned}
F_{\Omega} & =\frac{1}{\pi} \operatorname{Re}\left(\int_{\omega_{1}}^{\omega_{2}} \Psi(\imath \nu) \mathrm{d} \nu\right) \\
& =\operatorname{Re}\left(\frac{\imath}{\pi} \ln \left(\left(A+\imath \omega_{1} I_{n}\right)^{-1}\left(A+\imath \omega_{2} I_{n}\right)\right)\right)=\operatorname{Re}\left(\frac{\imath}{\pi} \ln \left(\mathcal{C}\left(A, \imath \omega_{1}, \imath \omega_{2}\right)\right)\right),
\end{aligned}
$$

where

$$
\mathcal{C}(A, \mu, \nu):=\left(A+\mu I_{n}\right)^{-1}\left(A+\nu I_{n}\right), \quad \mu, \nu \in \mathbb{C}, \mu \notin-\Lambda(A),
$$

denotes a generalized Cayley transformation of $A$ and $\ln (M)$ is the principal branch of the matrix-valued natural logarithm of $M$ with $\Lambda(M) \cap \mathbb{R}_{-}=\emptyset$.

Note that for frequency regions of the form $\Omega=[-\omega, \omega]$, it can be shown [46] that $(2.7 \mathrm{~b})$ simplifies to

$$
F_{\Omega}=\operatorname{Re}\left(\frac{\imath}{\pi} \ln \left(-A-\imath \omega I_{n}\right)\right)
$$

Copyright $@$ by SIAM. Unauthorized reproduction of this article is prohibited. 
Moreover, Theorems 2.2 and 2.3 can be generalized to multiple, concatenated frequency segments

$$
\Omega=\bigcup_{i=1}^{k}\left[-\omega_{2 i},-\omega_{2 i-1}\right] \cup\left[\omega_{2 i-1}, \omega_{2 i}\right] \quad \text { with } \quad 0 \leq \omega_{1}<\omega_{2}<\cdots<\omega_{2 k}<\infty,
$$

where the matrix-valued logarithm of a product of generalized Cayley transformations occurs; see [46, Corollary 3.1]. For simplification and brevity, we mainly focus on frequency restrictions of the form (2.3) in the remainder.

Computing $F_{\Omega}$ involves the evaluation of a function $f($.$) in A$ with $f(z)=\ln \frac{\lambda+\imath \omega_{2}}{\lambda+\imath \omega_{1}}$, i.e., the logarithm of a generalized Cayley transformation. For matrices of large dimensions, this appears to be a very formidable and expensive task. Some strategies that make an efficient treatment of $F_{\Omega}$ possible are proposed in section 4 . There, the numerical approximation of the frequency-limited Gramians by low-rank solutions $P_{\Omega} \approx Z_{P_{\Omega}} Z_{P_{\Omega}}^{T}, Q_{\Omega} \approx Z_{Q_{\Omega}} Z_{Q_{\Omega}}^{T}$ is also investigated. The eigenvalue decay of $P_{\Omega}, Q_{\Omega}$ and, consequently, how well they can be approximated by such low-rank solutions is considered in the next section. With the low-rank solution factors $Z_{P_{\Omega}}, Z_{Q_{\Omega}}$, FLBT can be carried out analogously to Algorithm 1.1 by substituting the CALEs (1.2) for the infinite Gramians by the frequency-limited CALEs (2.6) in line 1 and using $Z_{P_{\Omega}}$, $Z_{Q_{\Omega}}$ in the remaining steps. In contrast to BT without frequency restrictions, FLBT is not guaranteed to preserve the stability of (1.1) such that also no error bound can be given. We briefly come back to this issue in section 5.2.

3. On the eigenvalue decay of the frequency-limited Gramians. We expect that the frequency-limited Gramians $P_{\Omega}, Q_{\Omega}$ in (2.6) can be well approximated by low-rank solutions because their inhomogeneities are of low rank $2 m, 2 p \ll n$. Comparing the infinite CALEs (1.2) with the frequency-limited ones (2.6), these inhomogeneities are the only differences in (2.6). Recalling the theory on the existence of low-rank solutions of matrix equations [45, 1, 22, 55], the rank of the inhomogeneity of a matrix equation is an influential factor on the numerical rank of the solution. The rank of the inhomogeneities in (2.6) is twice as large as the rank of the inhomogeneities $B B^{T}$ and $C^{T} C$ of the CALEs (1.2) for the infinite Gramians. Thus, one is tempted to expect that the numerical rank of $P_{\Omega}, Q_{\Omega}$ is larger than the numerical rank of $P$, $Q$. Observations in practice, however, often show the exact opposite phenomenon, i.e., $P_{\Omega}, Q_{\Omega}$ are of smaller numerical rank than $P, Q$.

On the one hand, this seems to be counterintuitive as the coefficient matrices in both (1.2) and (2.6) are the same. On the other hand, comparing (2.1) and (2.2), it appears intuitively clear that $P_{\Omega}, Q_{\Omega}$ have smaller numerical rank since the integration range is smaller such that less information enters the integrals. A general approach for investigating the numerical rank of solutions of matrix equations is to look at the eigenvalue decay of the solutions. As for the unlimited Gramians, however, obtaining a general analytic prediction on the exact eigenvalue decay is difficult. Motivated by the approaches used in $[1,55]$, we try to bound the eigenvalues of $P$ and $P_{\Omega}$ in the following. To this end, we restrict to the infinite and frequency-limited reachability Gramians $P, P_{\Omega}$ in the case $m=1$, i.e., $B=b \in \mathbb{R}^{n}$. The observability Gramians can be dealt with similarly and generalizations to the case $m>1$ can be drawn from, e.g, [1]. The next lemma provides useful factorizations of $P$ and $P_{\Omega}$.

LEMma 3.1 (factorization of $P$ and $P_{\Omega}$ ). Let $A$ in (1.2) and (2.6a) be diagonalizable, i.e., there exists a nonsingular matrix $X \in \mathbb{C}^{n \times n}$ such that $A=X \Lambda X^{-1}$ with $\Lambda=\operatorname{diag}\left(\lambda_{1}, \ldots, \lambda_{n}\right), \lambda_{i} \in \Lambda(A)$. Furthermore, assume that $(A, b)$ is controllable. 
a) The reachability Gramian $P$ can be expressed as

$$
P=X_{b} K X_{b}^{H} \quad \text { with } \quad X_{b}:=X \operatorname{diag}\left(X^{-1} b\right) .
$$

The matrix $K=\left(\frac{-1}{\lambda_{i}+\overline{\lambda_{j}}}\right)_{i, j=1}^{n}$ is a Hermitian positive definite Cauchy matrix.

b) The frequency-limited reachability Gramian $P_{\Omega}$ can be factored as

$$
P_{\Omega}=X_{b} K_{\Omega} X_{b}^{H}, \quad \text { where } K_{\Omega}:=\Gamma K+K \Gamma^{H}
$$

with $\Gamma=X^{-1} F_{\Omega} X=\operatorname{diag}\left(\gamma_{1}, \ldots, \gamma_{n}\right)$. The matrix $K_{\Omega}$ is a Hermitian positive definite Loewner matrix.

Proof. Result a) is established in [1, Lemma 3.2].

For b), the expression (2.4) reveals

$$
\begin{aligned}
P_{\Omega}=F_{\Omega} P+P F_{\Omega}^{H} & =X \Gamma X^{-1} X_{b} K X_{b}^{H}+X_{b} K X_{b}^{H} X^{-H} \Gamma^{H} X^{H} \\
& =X \Gamma \operatorname{diag}\left(X^{-1} b\right) K X_{b}^{H}+X_{b} K \operatorname{diag}\left(X^{-1} b\right)^{H} \Gamma^{H} X^{H},
\end{aligned}
$$

from which the factorization follows since diagonal matrices commute. Upon closer inspection, $K_{\Omega}:=\Gamma K+K \Gamma^{H}=\left(-\frac{\gamma_{i}+\overline{\gamma_{j}}}{\lambda_{i}+\overline{\lambda_{j}}}\right)_{i, j=1}^{n}$ is obviously a Hermitian Loewner matrix [17], which inherits the positive definiteness from $P_{\Omega} \succ 0$.

For every product $F T L$ of three matrices $F, T, L$ of appropriate dimensions, there is the well-known result [30, Theorem 3.3.2] that $\sigma_{j}(F T L) \leq\|F\|\|L\| \sigma_{j}(T)$. Applying this to (3.1) and (3.2) yields

$$
\lambda_{j}^{\downarrow}(P) \leq \zeta \lambda_{j}^{\downarrow}(K), \quad \lambda_{j}^{\downarrow}\left(P_{\Omega}\right) \leq \zeta \lambda_{j}^{\downarrow}\left(K_{\Omega}\right)
$$

with $\zeta:=\left\|X_{b}\right\|^{2}$. Here, we use that for any Hermitian positive definite matrix, its eigenvalues in a decreasing order coincide with its singular values. Hence, the eigenvalues of $P$ and $P_{\Omega}$ are bounded by the eigenvalues of $K$ and $K_{\Omega}$, respectively. However, as $\left\|X_{b}\right\|$ can be arbitrarily large, e.g., when $A$ is nonnormal $(\kappa(X)>1)$, there might be a large deviation between $\lambda_{j}^{\downarrow}(P), \lambda_{j}^{\downarrow}\left(P_{\Omega}\right)$ and $\lambda_{j}^{\downarrow}(K), \lambda_{j}^{\downarrow}\left(K_{\Omega}\right)$. The effect of nonnormality to CALE solutions is investigated from a different perspective in [3]. At this point we stress that the focus of this section is not to give a precise estimation of the eigenvalue decay of the Gramians, but to relate $\lambda_{j}^{\downarrow}(P)$ to $\lambda_{j}^{\downarrow}\left(P_{\Omega}\right)$ in the sense that $\lambda_{j}^{\downarrow}\left(K_{\Omega}\right)$ can be bounded by $\lambda_{j}^{\downarrow}(K)$. For this, the following bound can be readily established.

LEMMA 3.2. For the eigenvalues of the matrices $K$ and $K_{\Omega}$ in (3.1) and (3.2), respectively, it holds for $j=1, \ldots, n$ that,

$$
\lambda_{j}^{\downarrow}\left(K_{\Omega}\right) \leq 2\left|\lambda_{j}^{\downarrow}(\Gamma)\right| \lambda_{j}^{\downarrow}(K) \leq 2 \rho(\Gamma) \lambda_{j}^{\downarrow}(K),
$$

and, thus, $\lambda_{j}^{\downarrow}\left(P_{\Omega}\right) \leq 2 \zeta\left|\gamma_{j}^{\downarrow}\right| \lambda_{j}^{\downarrow}(K)$.

Proof. Recall that for any matrix $M \in \mathbb{C}^{n \times n}$, its Hermitian part is $H_{M}:=$ $\frac{1}{2}\left(M+M^{H}\right)$. The eigenvalues of $H_{M}$ can be bounded by the singular values of $M$ by [30, Corollary 3.1.5]. Obviously, $K_{\Omega}$ is twice the Hermitian part of $N:=\Gamma K$, i.e., $K_{\Omega}=2 H_{N}=N+N^{H}$, and a straightforward application of the said corollary and [30, Theorem 3.3.2] yields $\lambda_{j}^{\downarrow}\left(K_{\Omega}\right) \leq 2 \sigma_{j}(N) \leq 2 \sigma_{j}(\Gamma) \sigma_{j}(K)$, which leads to the result since $\Gamma$ is diagonal and $K$ Hermitian positive definite.

This result should by no means be understood as very accurate because [30, Corollary 3.1.5] introduces a large overestimation regarding the magnitudes of $\lambda_{j}^{\downarrow}\left(K_{\Omega}\right)$. To

Copyright (C) by SIAM. Unauthorized reproduction of this article is prohibited. 
the authors' knowledge, there is no tighter bound for the eigenvalues of the Hermitian part of a matrix available. Nonetheless, Lemma 3.2 reveals that the spectral radius $\rho(\Gamma)=\rho\left(F_{\Omega}\right)$ has a large influence on the eigenvalues of $K_{\Omega}$. In the next lemma, we derive insightful bounds for the eigenvalues and spectral radius of $F_{\Omega}$.

LEMMA 3.3 (eigenvalue bounds for $F_{\Omega}$ ). Let A satisfy the same assumptions as above and let

$$
\hat{\rho}:=\left|\lambda_{q}\right|, \quad \hat{\eta}:=\left|\operatorname{Im}\left(\lambda_{q}\right)\right|, \quad \lambda_{q}:=\underset{\lambda \in \lambda(A)}{\operatorname{argmax}}\left|\frac{\operatorname{Im}(\lambda)}{\operatorname{Re}(\lambda)}\right| .
$$

Then $F_{\Omega}$ is nonsingular and for $\gamma_{j} \in \Lambda\left(F_{\Omega}\right), j=1, \ldots, n$, it holds that

$$
\begin{aligned}
0<\operatorname{Re}\left(\gamma_{j}\right) & <\frac{1}{2}, \\
\left|\operatorname{Im}\left(\gamma_{j}\right)\right| & <\frac{1}{4 \pi} \ln \frac{\hat{\rho}+\hat{\hat{\rho}}}{\hat{\rho}-\hat{\eta}}=: \chi
\end{aligned}
$$

and, consequently, $\rho\left(F_{\Omega}\right)=\rho(\Gamma)<\frac{1}{2} \sqrt{1+4 \chi^{2}}$.

Proof. Since $A$ is assumed to be diagonalizable, we have

$$
\begin{aligned}
F_{\Omega} & =\operatorname{Re}\left(\frac{\imath}{\pi} \ln \left(\mathcal{C}\left(A, \imath \omega_{1}, \imath \omega_{2}\right)\right)\right)=\operatorname{Re}\left(\frac{\imath}{\pi} X \ln \operatorname{diag}\left(\theta_{1}, \ldots, \theta_{n}\right) X^{-1}\right) \\
& =\operatorname{Re}\left(X \operatorname{diag}\left(\hat{\gamma}_{1}, \ldots, \hat{\gamma}_{n}\right) X^{-1}\right)
\end{aligned}
$$

where

$$
\hat{\gamma}_{j}:=\frac{\imath}{\pi}\left(\ln \left|\theta_{j}\right|+\imath \arg \theta_{j}\right), \quad \theta_{j}=\frac{\lambda_{j}+\imath \omega_{2}}{\lambda_{j}+\imath \omega_{1}} \in \Lambda\left(\mathcal{C}\left(A, \imath \omega_{1}, \imath \omega_{2}\right)\right), \quad \lambda_{j} \in \Lambda(A) .
$$

Since $\theta_{j} \neq 1$ it holds that $\hat{\gamma}_{j} \neq 0 \forall j$, which proves the nonsingularity of $F_{\Omega}$. Furthermore, the eigenpairs of $A$ occur either in the form $\left(\lambda_{j}, x_{j}\right) \in \mathbb{R}_{-} \times \mathbb{R}^{n}$ or as two complex conjugate pairs $\left(\lambda_{j}, x_{j}\right),\left(\lambda_{j+1}=\bar{\lambda}_{j}, x_{j+1}=\bar{x}_{j}\right) \in \mathbb{C}_{-} \times \mathbb{C}^{n}$. Hence, there exists a block-diagonal, nonsingular matrix $T=\operatorname{diag}\left(T_{1}, \ldots, T_{n}\right)$ with $T_{j}=1$ if $\lambda \in \mathbb{R}_{-}$, and $T_{j}=\left[\begin{array}{cc}1 & -\imath \\ 1 & \imath\end{array}\right]$ if $\lambda_{j} \in \mathbb{C}_{-}$, such that $X_{\mathbb{R}}:=X T \in \mathbb{R}^{n \times n}$ and, hence,

$$
F_{\Omega}=X_{\mathbb{R}} \operatorname{Re}\left(T^{-1} \operatorname{diag}\left(\hat{\gamma}_{1}, \ldots, \hat{\gamma}_{n}\right) T\right) X_{\mathbb{R}}^{-1} .
$$

Let us at first investigate the case of real eigenvalues $\lambda_{j}$ for which $T_{j}=1$ such that the diagonal entries above are

$$
\hat{\gamma}_{j}=\gamma_{j}:=\operatorname{Re}\left(\hat{\gamma}_{j}\right)=\frac{-\arg \theta_{j}}{\pi} .
$$

Since

$$
\theta_{j}=\frac{\lambda_{j}^{2}+\omega_{1} \omega_{2}}{\lambda_{j}^{2}+\omega_{1}^{2}}+\imath \frac{\left(\omega_{2}-\omega_{1}\right) \lambda_{j}}{\lambda_{j}^{2}+\omega_{1}^{2}},
$$

we have $\operatorname{Re}\left(\theta_{j}\right)>0$ and $\operatorname{Im}\left(\theta_{j}\right)<0$ such that $-\frac{\pi}{2}<\arg \theta_{j}<0$, which yields the desired result (3.4a), as well as obviously (3.4b).

For each complex conjugate pair of eigenvalues $\lambda_{j}, \overline{\lambda_{j}}$ the associated $2 \times 2$ block in $(3.5)$ is

Copyright $@$ by SIAM. Unauthorized reproduction of this article is prohibited. 


$$
\begin{aligned}
\operatorname{Re}\left(T_{j}^{-1} \operatorname{diag}\left(\hat{\gamma}_{j}, \hat{\gamma}_{j+1}\right) T_{j}\right) & =\frac{1}{2} \operatorname{Re}\left(\left[\begin{array}{cc}
1 & 1 \\
\imath & -\imath
\end{array}\right]\left[\begin{array}{cc}
\hat{\gamma}_{j} & 0 \\
0 & \hat{\gamma}_{j+1}
\end{array}\right]\left[\begin{array}{cc}
1 & -\imath \\
1 & \imath
\end{array}\right]\right) \\
& =\frac{1}{2} \operatorname{Re}\left(\left[\begin{array}{cc}
\hat{\gamma}_{j}+\hat{\gamma}_{j+1} & -\imath\left(\hat{\gamma}_{j}-\hat{\gamma}_{j+1}\right) \\
\imath\left(\hat{\gamma}_{j}-\hat{\gamma}_{j+1}\right) & \hat{\gamma}_{j}+\hat{\gamma}_{j+1}
\end{array}\right]\right) \\
& =\frac{1}{2 \pi}\left[\begin{array}{cc}
-\arg \theta_{j}-\arg \theta_{j+1} & \ln \left|\theta_{j}\right|-\ln \left|\theta_{j+1}\right| \\
-\ln \left|\theta_{j}\right|+\ln \left|\theta_{j+1}\right|-\arg \theta_{j}-\arg \theta_{j+1}
\end{array}\right] \\
& =\frac{1}{2 \pi}\left[\begin{array}{cc}
-\arg \theta_{j} \cdot \theta_{j+1} & \ln \left|\theta_{j} / \theta_{j+1}\right| \\
-\ln \left|\theta_{j} / \theta_{j+1}\right|-\arg \theta_{j} \cdot \theta_{j+1}
\end{array}\right] .
\end{aligned}
$$

Hence, $F_{\Omega}$ has the eigenvalues

$$
\left\{\gamma_{j}, \gamma_{j+1}=\overline{\gamma_{j}}\right\}=\left\{\frac{-1}{2 \pi}\left(\arg \left(\theta_{j} \cdot \theta_{j+1}\right) \mp \imath \ln \left|\theta_{j} / \theta_{j+1}\right|\right)\right\}
$$

corresponding to each complex pair of eigenvalues $\left\{\lambda_{j}, \overline{\lambda_{j}}\right\} \subset \Lambda(A)$. It holds that

$$
\begin{gathered}
\theta_{j} \theta_{j+1}=\frac{1}{z}\left(\left(\left|\lambda_{j}\right|^{2}-\omega_{2}^{2}\right)\left(\left|\lambda_{j}\right|^{2}-\omega_{1}^{2}\right)+4 \omega_{1} \omega_{2} \operatorname{Re}\left(\lambda_{j}\right)^{2}\right. \\
\left.+\imath 2 \operatorname{Re}\left(\lambda_{j}\right)\left(\left|\lambda_{j}\right|^{2}+\omega_{1} \omega_{2}\right)\left(\omega_{2}-\omega_{1}\right)\right)
\end{gathered}
$$

with $z:=\left(\left|\lambda_{j}\right|^{2}-\omega_{1}^{2}\right)^{2}+4 \omega_{1}^{2} \operatorname{Re}\left(\lambda_{j}\right)^{2}$ and we find $\operatorname{Im}\left(\theta_{j} \theta_{j+1}\right)<0$ such that $-\pi<$ $\arg \theta_{j} \theta_{j+1}<0$ from which $\operatorname{Re}\left(\gamma_{j}\right)=\operatorname{Re}\left(\overline{\gamma_{j}}\right)=-\frac{1}{2 \pi}\left(\arg \theta_{j} \theta_{j+1}\right)<\frac{1}{2}$ follows. For the imaginary parts of $\gamma_{j}$ assume without loss of generality $\operatorname{Im}\left(\lambda_{j}\right)>0$ and consider

$$
0<\Theta_{j}:=\left|\frac{\theta_{j}}{\theta_{j+1}}\right|^{2}=\left(\frac{\left|\lambda_{j}\right|^{2}+\omega_{1}^{2}-2 \operatorname{Im}\left(\lambda_{j}\right) \omega_{1}}{\left|\lambda_{j}\right|^{2}+\omega_{1}^{2}+2 \operatorname{Im}\left(\lambda_{j}\right) \omega_{1}}\right)\left(\frac{\left|\lambda_{j}\right|^{2}+\omega_{2}^{2}+2 \operatorname{Im}\left(\lambda_{j}\right) \omega_{2}}{\left|\lambda_{j}\right|^{2}+\omega_{2}^{2}-2 \operatorname{Im}\left(\lambda_{j}\right) \omega_{2}}\right)=\zeta_{1} \zeta_{2}
$$

with $0<\zeta_{1} \leq 1<\zeta_{2}$. Hence,

$$
\left|\ln \Theta_{j}\right|<\max \left(\ln \max \Theta_{j},\left|\ln \min \Theta_{j}\right|\right) .
$$

Now, $\zeta_{2}$ is, for a fixed $\lambda_{j}$, maximal if $\omega_{2}=\left|\lambda_{j}\right|$. In that case

$$
\zeta_{2}=\frac{\left|\lambda_{j}\right|+\operatorname{Im}\left(\lambda_{j}\right)}{\left|\lambda_{j}\right|-\operatorname{Im}\left(\lambda_{j}\right)}=\frac{\sqrt{1+q_{j}^{2}}+q_{j}}{\sqrt{1+q_{j}^{2}}-q_{j}}, \quad q_{j}:=\frac{\operatorname{Im}\left(\lambda_{j}\right)}{\left|\operatorname{Re}\left(\lambda_{j}\right)\right|},
$$

which increases as $q_{j}$ increases and, hence, the maximum value of $\zeta_{2}$ is attained at $q_{\max }=\frac{\operatorname{Im}\left(\lambda_{q}\right)}{\left|\operatorname{Re}\left(\lambda_{q}\right)\right|}$, i.e., for $\lambda_{q}$. Using also $\max \zeta_{1}=1$ (attained at $\omega_{1}=0$ ) yields

$$
\max \Theta_{j}<\left(\frac{\hat{\rho}+\hat{\eta}}{\hat{\rho}-\hat{\eta}}\right) .
$$

Furthermore, $\min \zeta_{2}=1$ and, for a fixed $\lambda_{j}, \zeta_{1}$ is by a similar reasoning minimal if $\omega_{1}=\left|\lambda_{q}\right|$. This leads finally to

$$
\left|\ln \Theta_{j}\right|<\max \left(\ln \frac{\hat{\rho}+\hat{\eta}}{\hat{\rho}-\hat{\eta}},\left|\ln \frac{\hat{\rho}-\hat{\eta}}{\hat{\rho}+\hat{\eta}}\right|\right)
$$

from which (3.4b) follows. $\quad \mathrm{a}$

Because the proof deals with the real and imaginary parts of the $\gamma_{i}$ independently, the results represent upper bounds for the largest attainable real, imaginary part and spectral radius. Also, if $\omega_{1}=0$, the bound for $\rho(\Gamma)$ can be slightly altered to $\rho(\Gamma)<\frac{1}{4} \sqrt{1+16 \chi^{2}}$. Together with Lemma 3.2 these bounds reveal how the spectral 
radius of $\Gamma$ influences the eigenvalues of $K_{\Omega}$. In particular, we can deduce possibilities when the $\lambda_{j}^{\downarrow}\left(K_{\Omega}\right)$ are significantly smaller than the $\lambda_{j}^{\downarrow}(K)$. For matrices $A$ with real spectra, $F_{\Omega}$ also has only real eigenvalues with $0<\gamma_{j}<\frac{1}{2}$ by Lemma 3.3. Thus, $\Gamma \succ 0$ such that the bound in Lemma 3.2 becomes $\lambda_{j}^{\downarrow}\left(K_{\Omega}\right) \leq 2 \gamma_{j}^{\downarrow} \lambda_{j}^{\downarrow}(K)$ for $j=1, \ldots, n$. Obviously, if the considered frequency interval is small, i.e., $\omega_{2}-\omega_{1}$ is small compared to $\frac{\lambda_{j}}{\lambda_{j}^{2}+\omega_{1} \omega_{2}}$, then $\operatorname{Im}\left(\theta_{j}\right)$ will be close to zero and so will $\arg \theta_{j}$.

If $\Lambda(A)$ has complex eigenvalues the situation is considerable more subtle, but the proof of Lemma 3.3 already indicates that setting the interval limits $\omega_{1}, \omega_{2}$ equal or close to absolute values of eigenvalues of $A$ can increase the spectral radius $\rho\left(F_{\Omega}\right)$. In particular, setting the interval limits close to $\left|\lambda_{q}\right|$ will lead to the largest values of $\chi$ in $(3.4 \mathrm{~b})$ and, thus, to large spectral radii. This is observed in numerical experiments but, even if $\rho\left(F_{\Omega}\right)>\frac{1}{2}$, the eigenvalues of $K_{\Omega}$ seem to be never much greater than the ones of $P$.

To conclude, we expect that the values of $\lambda_{j}^{\downarrow}\left(K_{\Omega}\right)$ will be noticeably smaller than $\lambda_{j}^{\downarrow}(K)$ if the chosen interval $\left[\omega_{1}, \omega_{2}\right]$ is small compared to the spectral radius of $A$ and if the interval boundaries $\omega_{1}, \omega_{2}$ are not close to the magnitude of the eigenvalues of $A$ whose imaginary parts dominate their real parts. This argumentation will also carry over to $\lambda_{j}^{\downarrow}\left(P_{\Omega}\right)$ and $\lambda_{j}^{\downarrow}(P)$ by (3.3).

The main conclusion of this section is that although

$$
\operatorname{rank}\left(F_{\Omega} B B^{T}+B B_{\Omega}^{T} F_{\Omega}^{T}\right)=2 \operatorname{rank}\left(B B^{T}\right),
$$

under reasonable and quantifiable assumptions on the interval $\left[\omega_{1}, \omega_{2}\right]$, the eigenvalues of $P_{\Omega}$ decay at a faster rate than those of $P$. Hence, $P_{\Omega}$ has a smaller numerical rank than $P$. Consequently, we can expect that there exist low-rank solution factors $Z_{P}$ and $Z_{P_{\Omega}}$ leading to low-rank solutions of comparable accuracy in the sense

$$
\left\|P-Z_{P} Z_{P}^{H}\right\| \approx\left\|P_{\Omega}-Z_{P_{\Omega}} Z_{P_{\Omega}}^{H}\right\|
$$

but with $\operatorname{rank}\left(Z_{P}\right) \geq \operatorname{rank}\left(Z_{P_{\Omega}}\right)$. This is also confirmed by our numerical experiments. The same holds trivially also for low-rank approximation of $Q$ and $Q_{\Omega}$. Moreover, it will turn out later that in some cases computing $Z_{P_{\Omega}}, Z_{Q_{\Omega}}$ is less costly than computing $Z_{P}, Z_{Q}$, which can even make FLBT numerically cheaper than the standard (unlimited) BT. Algorithms for computing the low-rank solution factors are the topic of the next section.

Remark 3.4.

(a) For the standard CALEs (1.2), the above considerations of Lemma 3.1 are continued in [1], where a square-root free Cholesky factorization of the form $K=L \Delta L^{H}$ with a unit lower triangular $L$ and $\Delta=\operatorname{diag}\left(\delta^{(1)}, \ldots, \delta^{(n)}\right) \succ 0$ is used. There are explicit formulas [20] for the diagonal entries $\delta^{(i)}$, which appear to decay to zero at a similar rate as the eigenvalues of $P$, especially if $A$ is not too far from normal. A square-root free Cholesky factorization also exists for the frequency-limited Gramian: $K_{\Omega}=L_{\Omega} \Delta_{\Omega} L_{\Omega}^{H}$ with $\Delta_{\Omega}=$ $\operatorname{diag}\left(\delta_{\Omega}^{(1)}, \ldots, \delta_{\Omega}^{(n)}\right) \succ 0$. A basic Cholesky algorithm [21] can be employed to find how the entries of $L_{\Omega}$ and $\Delta_{\Omega}$ are built from the entries of $L, \Delta$, and $\Gamma$. It is easy to show that $\delta_{\Omega}^{(1)}=2 \operatorname{Re}\left(\gamma_{1}\right) \delta^{(1)}<\delta^{(1)}$, but the calculations for the remaining entries become very tedious and lengthy such that we do not report them here for the sake of brevity.

(b) In [22], another well-known theoretical result regarding the existence of lowrank solutions of large matrix equations is proposed. Following [22], low-rank 
solutions of $P$ and $P_{\Omega}$ are given by $P_{k}:=\sum_{i=-k}^{k} \xi_{i} B_{i} B_{i}^{T}, B_{i}:=\exp \left(t_{i} A\right) B$ and $P_{\Omega, k}:=\sum_{i=-k}^{k} \xi_{i} \tilde{B}_{i} F_{m} \tilde{B}_{i}^{T}, \tilde{B}_{i}:=\exp \left(t_{i} A\right)\left[B, B_{\Omega}\right]$, respectively, with the flipping matrix

$$
F_{h}:=\left[\begin{array}{ll}
0 & 1 \\
1 & 0
\end{array}\right] \otimes I_{h}
$$

The constants $\xi_{i}, t_{i}$ are quadrature weights and nodes not important for the discussion here. Moreover, the approximation errors can be bounded by

$$
\left\|P-P_{k}\right\| \leq \phi \pi^{-1}\|B\|^{2}, \quad\left\|P_{\Omega}-P_{\Omega, k}\right\| \leq \phi \pi^{-1}\left\|\left[B, B_{\Omega}\right] F_{m}\left[B, B_{\Omega}\right]^{T}\right\|,
$$

where the constant $\phi$ depends entirely on $A$. Obviously, the bound $\operatorname{rank}\left(P_{\Omega, k}\right) \leq 2(k+1) m$ is larger than $\operatorname{rank}\left(P_{k}\right) \leq(k+1) m$ and the above error bounds differ only by the norms of the inhomogeneities of the CALEs (1.2), (2.6a). Notice that the difference in the decay rates of the eigenvalues of $P$ and $P_{\Omega}$ also can be observed if the inhomogeneities would be scaled to unit norm. Hence, this approach offers no useful explanation for why $P_{\Omega}$ can in practice often be approximated by low-rank solutions of smaller rank compared to $P$.

4. Numerical methods for computing the low-rank approximations. Motivated by the expected low numerical rank of $P_{\Omega}, Q_{\Omega}$, we aim at computing, as in standard BT, low-rank approximations $P_{\Omega} \approx Z_{P_{\Omega}} Z_{P_{\Omega}}^{T}, Q_{\Omega} \approx Z_{Q_{\Omega}} Z_{Q_{\Omega}}^{T}$ with $Z_{P_{\Omega}} \in \mathbb{R}^{n \times r_{1}}, Z_{Q_{\Omega}} \in \mathbb{R}^{n \times r_{2}}, r_{1}, r_{2} \ll n$. Before the frequency-limited CALEs can be approached by numerical methods, which compute low-rank solution factors, the matrix $F_{\Omega}$ has to be treated. This is the subject of the next subsection. After that, some strategies for computing the low-rank solution factors $Z_{P_{\Omega}}, Z_{Q_{\Omega}}$ will be discussed.

4.1. Dealing with the matrix-valued logarithm. In FLBT, the matrix $F_{\Omega}$ requires the evaluation of a matrix-valued function $f$ in $A$. Most state-of-the-art algorithms for that purpose work, e.g., with the Schur form of $A$ and additional matrix multiplications [28]. In our situation, $f(A)=\operatorname{Re}\left(\frac{\imath}{\pi} \ln \mathcal{C}\left(A, \imath \omega_{1}, \imath \omega_{2}\right)\right)$ such that the Schur form of $\mathcal{C}\left(A, \imath \omega_{1}, \imath \omega_{2}\right)$ can be deduced from the Schur form of $A$. For the matrix-valued logarithm, a very robust and often applied method is the inverse scaling and squaring algorithm [28, Chapter 11] and its variants. The method is called by the MATLAB routine logm. However, as computing the Schur form has a cubic complexity and quadratic memory demands, these approaches are not feasible for the large-scale case we are interested in.

If we plan to obtain the frequency-limited Gramians $P_{\Omega}, Q_{\Omega}$ from solving the frequency-limited CALEs (2.6), we observe that only

$$
\begin{aligned}
B_{\Omega} & :=F_{\Omega} B=f(A) B \\
\text { and } \quad C_{\Omega}: & =C F_{\Omega}=C f(A)
\end{aligned}
$$

are required for setting up the inhomogeneities in (2.6). Hence, only the products $f(A) B$ and $f(A)^{H} C^{T}$, i.e., $m$ and $p$ matrix-vector products with $f(A)$ and $f(A)^{H}$, respectively, are needed. Although $f(A)$ is still involved, computing the matrix-vector products of the form

$$
w=f(A) v, \quad w, v \in \mathbb{C}^{n}
$$


constitutes a much more attractive problem to overcome, even for large matrices $A$; see, for instance, [28, Chapter 13], [35, 10, 18, 36, 27], and the references therein, which provide several efficient numerical methods for this task.

Remark 4.1. The computation of the frequency-limited reachability Gramians $P_{\Omega}$ by (2.4) and using a low-rank solution factor $Z_{P}$ of the ordinary Gramian $P$ can also be reduced to the problem of computing the matrix-vector products of $f(A)$ with $Z_{P} \in \mathbb{R}^{n \times k_{P}}$ and similarly the problem of computing $Q_{\Omega}$ by (2.4) using $f(A)^{H} Z_{Q} \in$ $\mathbb{R}^{n \times k_{Q}}$. This approach is in general more expensive than using (4.1) and subsequently solving (2.6) because, in general, $k_{P}>m$ and $k_{Q}>p$, such that significantly more matrix-vector products with $f(A)$ would be required. In the following we discuss some approaches for (4.2) and their applicability for FLBT.

4.1.1. Quadrature based approaches. Recall that $F_{\Omega}$ was at first defined as the integral (2.5), which simplifies to (2.7a). Taking $B_{\Omega}=F_{\Omega} B$ as an example, an intuitive idea is to approximate (5.6) by means of a quadrature rule, i.e.,

$$
B_{\Omega}=\frac{1}{\pi} \operatorname{Re}\left(\int_{\omega_{1}}^{\omega_{2}}\left(\imath \nu I_{n}-A\right)^{-1} B \mathrm{~d} \nu\right) \approx \frac{1}{\pi} \operatorname{Re}\left(\sum_{k=1}^{h} \zeta_{k}\left(\imath \nu_{k} I_{n}-A\right)^{-1} B\right)
$$

using quadrature nodes $\nu_{k} \in\left[\omega_{1}, \omega_{2}\right], k=1, \ldots, h$, and weights $\zeta_{k}$ whose choice depends on the selected quadrature rule. In principle, any quadrature rule can be applied, where for reasons of accuracy, as well as efficiency, a method using an adaptive selection of nodes and weights is typically chosen. The integral command in MATLAB, e.g., employs the adaptive Gauss-Kronrod quadrature [34] and will be used in our numerical examples. Using (4.3) requires the solution of $h$ shifted linear systems with $m$ right-hand sides, which might easily become expensive, depending on the number of quadrature nodes. In [31], numerical quadrature is applied directly to the integrals (5.6) to obtain low-rank solution factors of $P_{\Omega}, Q_{\Omega}$. The POD approach in [16] is analogous.

4.1.2. Projection type methods. A further, frequently successfully applied and investigated approach for the matrix function times vector problem (4.2) is to use projections onto low-dimensional subspaces. Let $\mathcal{Q} \subset \mathbb{C}^{n}$ be a subspace with $\operatorname{dim} \mathcal{Q}=$ $k \ll n$ and let $Q_{k}=\left[q_{1}, \ldots, q_{k}\right] \in \mathbb{C}^{n \times k}$ with $\left\{q_{1}, \ldots, q_{k}\right\}$ being an orthonormal basis of $\mathcal{Q}$. Typically, $v \in \mathcal{Q}$ such that $v=Q_{k} \tilde{v}_{k}$ with $\tilde{v}_{k}:=Q_{k}^{H} v \in \mathbb{C}^{k}$. Approximating $w$ by its orthogonal projection onto $\mathcal{Q}$ gives

$$
w \approx Q_{k} Q_{k}^{H} w=Q_{k} \tilde{w} \in \mathcal{Q}, \quad \tilde{w}_{k}:=Q_{k}^{H} w \in \mathbb{C}^{k} .
$$

Imposing a Ritz-Galerkin orthogonality condition on the error of this approximation yields

$$
Q_{k} \tilde{w}_{k}-f(A) Q_{k} \tilde{v}_{k} \quad \perp \quad \mathcal{Q} \quad \Leftrightarrow \quad \tilde{w}_{k}=Q_{k}^{H} f(A) Q_{k} \tilde{v}_{k} \approx f\left(T_{k}\right) \tilde{v}_{k}
$$

with $T_{k}:=Q_{k}^{H} A Q_{k}$. The matrix $T_{k}$ is also called restriction of $A$ onto $\mathcal{Q}$. Hence, the approximate result is obtained by

$$
w_{k}:=Q_{k} f\left(T_{k}\right) Q_{k}^{H} v .
$$

Due to the small size of $T_{k} \in \mathbb{C}^{k \times k}$, the computation of $f\left(T_{k}\right)$ can then be carried out using methods for small, dense problems, e.g., the inverse scaling and squaring method discussed earlier in this section for our particular application. The quality of 
the approximation $w_{k}$ depends on how well $Q_{k} f\left(T_{k}\right) Q_{k}^{H} v$ approximates $f(A) v$, which, for general matrices $A$ and functions $f$, is difficult to predict. The above projection framework is usually carried out in an iterative manner using a sequence of nested subspaces $\mathcal{Q}_{1} \subseteq \mathcal{Q}_{2} \subseteq \cdots \subseteq \mathcal{Q}_{k} \subseteq \mathcal{Q}_{k+1}$ with increasing dimensions. To stop this iteration, we employ the simple stopping criterion

$$
\left\|w_{k}-w_{k-1}\right\| \leq \tau_{f}\left\|w_{k}\right\| .
$$

For certain choices of $\mathcal{Q}$, special matrices, and functions, more advanced, specially tailored stopping tests as well as error bounds can be found, e.g., in [28, Chapter 13.2], [18, 36, 27, 33]. The subspace $\mathcal{Q}$, or more precisely the sequence of subspaces, can be constructed in different ways and we restrict ourselves to the most common ones.

Setting up the subspace $\mathcal{Q}$ as standard Krylov subspace

$$
\mathcal{K}_{k}(A, v)=\operatorname{span}\left\{v, A v, A^{2} v, \ldots, A^{k-1} v\right\}
$$

is used for the approximation of (4.2) in, e.g., [35, 49]. The construction of the matrices $Q_{k}, T_{k}$ is usually done by the Lanczos or the Arnoldi algorithm [21] for Hermitian and, respectively, non-Hermitian $A$. It can be shown that with $\mathcal{K}_{k}$, the function $f$ is approximated by a polynomial $p_{k-1}$ of degree at most $k-1$ whose roots are the eigenvalues of $T_{k}$. To obtain a good approximation of (4.2), often large dimensions $k$ are needed, which makes this approach less practical.

Rational Krylov subspaces [12, 27] often provide much better approximations with smaller subspace dimensions $k$. They can be defined by

$$
\mathcal{K}_{k, \xi}^{\mathrm{rat}}(A, v)=d_{k-1}^{(\xi)}(A)^{-1} \mathcal{K}_{k}(A, v)
$$

with the denominator polynomial $d_{k-1}^{(\xi)}(z)=\prod_{j=1}^{k-1}\left(1-\frac{z}{\xi_{j}}\right)$ of degree $k-1$ having the poles $\xi_{1}, \ldots, \xi_{k-1} \in \mathbb{C} \cup\{\infty\}$. Hence, with $\mathcal{K}_{k}^{\text {rat }}$ the function $f$ is approximated by a rational function $r_{k}=p_{k-1} / d_{k-1}^{(\xi)}$. The poles $\xi$ of $r_{k}$ are typically referred to as shifts for the rational Krylov subspace. The orthonormal basis for $\mathcal{K}^{\text {rat }}$ is usually constructed with the rational Arnoldi algorithm [47] whose main numerical costs occur at solving linear systems of the form $(I-A / \xi) s=u$ for $s$.

The shifts are crucial for a fast convergence and good approximation results. A nice overview addressing various choices of a priori selected shifts for several functions $f$ can be found in [27]. In [12], an adaptive strategy is proposed, where the shift $\xi_{k+1}$ is computed from the data available after the rational Arnoldi iteration step $k$ is completed. The main idea is to consider the greedy method

$$
\xi_{k+1}=\underset{s \in \mathcal{D}_{k}}{\operatorname{argmax}}\left|r_{k}(s)\right|^{-1},
$$

where $\mathcal{D}_{k}$ is a set of discrete points from the boundary of the convex hull of $-\Lambda\left(T_{k}\right)$. We will use this adaptive shift generation in our numerical examples.

In our situation, since $f$ can be represented as integral (see (2.1)) of $\Psi(\imath \nu)$ within the integration domain $\Omega$, it appears reasonable to restrict the shifts to the imaginary region $\imath \Omega$. We therefore propose as modifications of the adaptive shift strategy to choose the set $\mathcal{D}_{k}$ a set of discrete points from $\imath \Omega$, i.e., $\mathcal{D}_{k} \subset \imath \Omega$. In most examples this leads to better results compared to the adaptive shifts based on the convex hull of $-\Lambda\left(T_{k}\right)$. 
A simplification of this approach is to use the shifts $\xi_{2 k}=\imath \omega_{1}$ and $\xi_{2 k-1}=\imath \omega_{2}$ alternatingly.

Choosing the shifts $\xi_{2 k}=\infty$ and $\xi_{2 k-1}=0$ in an alternating fashion yields the extended Krylov subspace:

$$
\mathcal{K}_{k}^{\text {ext }}(A, v)=\operatorname{span}\left\{v, A, \ldots, A^{k-1} v\right\} \cup \operatorname{span}\left\{A^{-1} v, \ldots A^{-k} v\right\},
$$

which is proposed in [11] and further investigated in [36]. In [52], an efficient algorithmic framework for constructing the basis and representation matrix $Q_{k}, T_{k}$ is established. One advantage of that choice is that the coefficient matrix of the linear system to solve does not change such that one can store and reuse a factorization of $A$ if direct solvers are applied, or recycle preconditioners and subspaces for iterative solvers. This can also be done if the shifts $\xi$ for $\mathcal{K}^{\text {rat }}$ are constant, which yields the shift-and-invert Krylov subspace $\mathcal{K}_{k}^{\mathrm{SI}}(A, v)[42,56]$. For the alternate use of $\imath \omega_{1}, \imath \omega_{2}$, two sparse matrix factorizations might be saved. We end this short introduction to Krylov subspace methods for $v=f(A) w$ with a number of comments regarding actual implementations.

Remark 4.2.

1. The matrices $Q_{k}, T_{k}$ in a basic implementation of the rational Arnoldi process are complex if some of the shifts $\xi$ are complex. Since in our setting the defining matrices $A, B, C$ are real, it is wise to construct a real basis for $\mathcal{K}^{\text {rat }}$, e.g., by using the modification to the rational Arnoldi process proposed in [48]. These changes basically amount to augment the basis matrix orthogonally by $\operatorname{Re}(s)$ and $\operatorname{Im}(s)$ for $s=(I-A / \xi)^{-1} u$ if $\xi \in \mathbb{C}$. This will also yield a real restriction $T_{k}$. Using this modification is, of course, reasonable because the number of complex arithmetic operations is reduced. In our application, since establishing the relation $(2.7 \mathrm{~b})$ from $(2.5)$ relied heavily on the fact that the matrix $A$ in $\Psi(\imath \nu)=(\imath \nu I-A)^{-1}$ is real, having a real $T_{k}$ also appears to be important if we want to safely employ (2.7b) to $T_{k}$. Issues with complex shifts are not present in methods using standard or extended Krylov subspaces.

2. If the number $m$ of columns in $B$ is larger than one, $m$ matrix-vector products with $f(A)$ are required. In this case, we utilize block versions [15] of the above Krylov subspaces, e.g.,

$$
\mathcal{K}_{k}(A, V)=\operatorname{span}\left\{V, A V, A^{2} V, \ldots, A^{k-1} V\right\}, \quad V \in \mathbb{C}^{n \times \ell} .
$$

Similar block versions of $\mathcal{K}^{\text {rat }}$ and $\mathcal{K}^{\text {ext }}$ can be easily deduced. Alternatives might be global Arnoldi methods [54] or, in the case of rational Krylov subspaces, tangential approaches [13]. There, the basis matrix is augmented by $s=(I-A / \xi)^{-1} U d$ for $U \in \mathbb{C}^{n \times m}$ and an appropriately chosen tangential direction vector $d \in \mathbb{C}^{m}$. This approach has also been applied in the context of $\mathcal{H}_{2}$ model order reduction [25]. A similar framework with respect to low-rank solutions of CALEs is presented in [57].

3. In our application we also need matrix-vector products $z=f(A)^{T} u$ of the transposed matrix function for computing $C_{\Omega}=C F_{\Omega}$. Thus, the above approaches also have to be applied using $A^{T}$ and $u$, leading to a subspace $\mathcal{Z}$ generated by $A^{T}$ and $u$, e.g.,

$$
\mathcal{Z}=\mathcal{K}_{k}\left(A^{T}, u\right)=\operatorname{span}\left\{u, A^{T} u, \ldots,\left(A^{T}\right)^{k-1}\right\}
$$

and the dual versions of $\mathcal{K}^{\text {rat }}, \mathcal{K}^{\text {ext }}$ are easily deduced. It is possible to utilize a two-sided Petrov-Galerkin condition [29] and construct $\mathcal{Z}$ bi-orthogonal to 
$\mathcal{Q}$. Both spaces can be generated simultaneously with the two-sided Lanczos process or modifications of it. Further details on generating dual subspaces $\mathcal{Q}, \mathcal{Z}$ can, e.g., be found in $[43,23,25]$.

4.2. Computing low-rank solution factors of the frequency-limited Gramians. Having computed $B_{\Omega}$ and $C_{\Omega}$, the frequency-limited CALEs (see also $(2.6))$

$$
\begin{aligned}
& A P_{\Omega}+P_{\Omega} A^{T}+B_{\Omega} B^{T}+B B_{\Omega}^{T}=0 \\
& A^{T} Q_{\Omega}+Q_{\Omega} A+C_{\Omega}^{T} C+C^{T} C_{\Omega}=0
\end{aligned}
$$

have to be solved for low-rank approximations. Any low-rank solution algorithm for large-scale CALEs can be employed here. In the following, we will discuss Krylov subspace methods as well as the low-rank alternating direction implicit (LR-ADI) iteration for this task. We will restrict ourselves to the treatment of the frequencylimited reachability CALE as the frequency-limited observability CALE can be dealt with in the same way.

4.2.1. Using Krylov subspace methods. In a similar way as we discussed in section 4.1.2, we can use a projection approach for solving the frequency-limited CALEs. Having a low-dimensional subspace $\mathcal{Q}_{k}=\operatorname{span}\left\{Q_{k}\right\}$ constructed, the lowrank solution is obtained as $P_{\Omega} \approx P_{\Omega, k}:=Q_{k} \tilde{P}_{\Omega, k} Q_{k}^{T}$, where $\tilde{P}_{\Omega, k}$ is the solution of the projected frequency-limited CALE

$$
T_{k} \tilde{P}_{\Omega, k}+\tilde{P}_{\Omega, k} T_{k}^{T}+\tilde{B}_{\Omega, k} \tilde{B}_{k}^{T}+\tilde{B}_{k} \tilde{B}_{\Omega, k}^{T}=0, \quad \tilde{B}_{k}:=Q_{k}^{T} B .
$$

Due to its small dimension, it can be solved by direct methods. Choices for $\mathcal{Q}_{k}$ include the same possibilities as for the matrix-function evaluations: standard [32], extended [52], as well as rational Krylov subspaces [12, 13], generated using $A, B$, and possibly a collection of (adaptively computed) shift parameters. If $P_{\Omega, k}$ is not accurate enough, the particular Krylov process used is continued.

Now assume $B_{\Omega}$ is approximated by such a projection approach, i.e., $B_{\Omega} \approx$ $B_{\Omega, k}:=Q_{k} \tilde{B}_{\Omega, k}$ with $\tilde{B}_{\Omega, k}:=f\left(T_{k}\right) Q_{k}^{T} B, T_{k}:=Q_{k}^{T} A Q_{k}$, where $Q_{k}$ is a real orthogonal matrix, which spans one of the aforementioned Krylov subspaces. In that case a nearby strategy is to reuse the information contained in the basis matrix $Q_{k}$ and continue the Krylov method for solving the frequency-limited CALE. In the majority of our numerical tests only very few additional iterations of the employed Krylov method were necessary to obtain the desired accuracy for $P_{\Omega}$ once an accurate $B_{\Omega, k}$ was found. Often, accurate approximations $B_{\Omega, k}$ and $P_{\Omega, k}$ were obtained in the same iteration step $k$, which makes this approach exceptionally efficient. Provided that $\tilde{P}_{\Omega, k} \geq 0$ in the projected equation (4.6), low-rank solution factors of $P_{\Omega}$ are given by $Z_{P_{\Omega}, k}=Q_{k} L_{k}$, where $L_{k}$ is a lower triangular Cholesky factor of $\tilde{P}_{\Omega, k}$. Alternatively, an eigendecomposition of $\tilde{P}_{\Omega, k}$ can be used which also enables a rank truncation; see, e.g., [52]. In Algorithm 4.1, we illustrate this strategy for approximating $B_{\Omega}$ and $P_{\Omega}$ in a single Krylov subspace algorithm.

In lines 1 and 12, orth should be understood as any stable (block) orthogonalization routine. By adjusting the basis generation in line 12 appropriately, any version of extended (EKSM), rational (RKSM), or adaptive rational Krylov subspace methods can be incorporated easily. The restriction $T_{j}$ in line 3 can be computed efficiently without additional matrix-vector products with $A$ by using relations developed in [52] and [47] for the EKSM and the RKSM, respectively. Typically, one has 


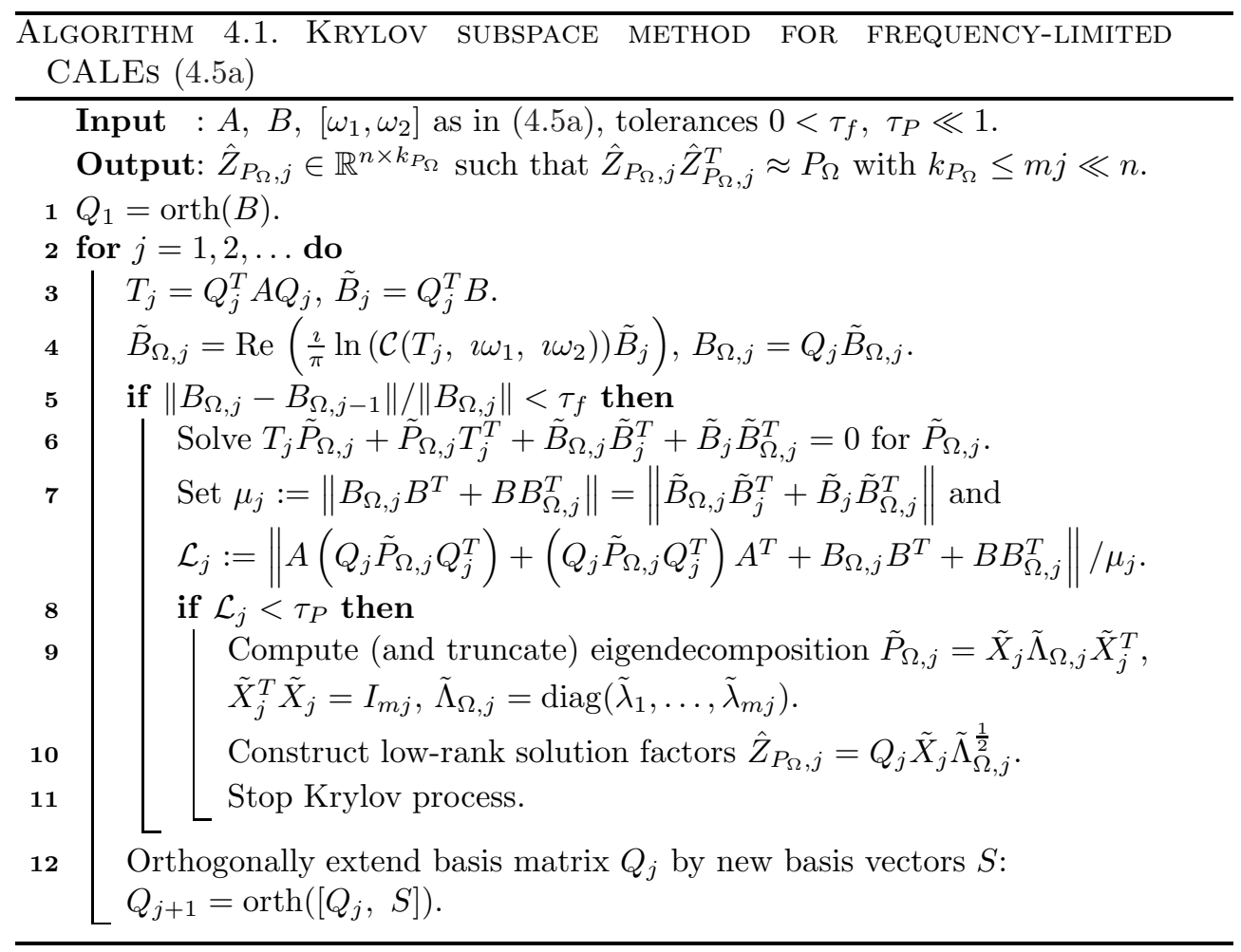

$\operatorname{span}\{B\} \subset \operatorname{span}\left\{Q_{j}\right\}$ and, thus, $\tilde{B}_{j}=Q_{j}^{T} B=\left[\beta^{T}, 0\right]^{T} \in \mathbb{R}^{m j \times m}$ with $\beta \in \mathbb{R}^{m \times m}$ such that the inhomogeneity of the projected CALE in line 6 is given by

$$
\begin{aligned}
\tilde{B}_{\Omega, j} \tilde{B}_{j}^{T}+\tilde{B}_{j} \tilde{B}_{\Omega, j}^{T} & =\left[\begin{array}{cc}
\tilde{\beta} \beta^{T}+\beta \tilde{\beta}^{T} & g^{T} \\
g & 0
\end{array}\right], \\
\text { where } g & :=\delta \beta^{T}, \quad \tilde{B}_{\Omega, j}=\left[\begin{array}{l}
\tilde{\beta} \\
\delta
\end{array}\right], \tilde{\beta} \in \mathbb{R}^{m \times m}, \delta \in \mathbb{R}^{(j-1) m \times m} .
\end{aligned}
$$

After $\left\|B_{\Omega, j}-B_{\Omega, j-1}\right\| /\left\|B_{\Omega, j}\right\|<\tau_{f}$ is achieved, one can also skip the computation of newer approximations $B_{\Omega, k}, k>j$, in the following iterations to save some computations in line 4 . In line 8, we employed a stopping criterion based on the scaled norm of the Lyapunov residual matrix. This norm can be computed efficiently without working with matrices $A, B, B_{\Omega, j}, Q_{j}$ of leading dimension $n$ [52]. If the RKSM with the adaptive, imaginary shifts has been used to approximate $B_{\Omega, j}$, one should switch to the convex hull based adaptive shifts [12] in the iterations steps for computing the low-rank solution factor of the CALE.

A potential weakness of Algorithm 4.1 and, in fact, of all Krylov subspace methods for CALEs is when $A+A^{T}$ is not negative definite (i.e., $A$ is not dissipative) because it can happen that $T_{j}$ has unstable eigenvalues such that the projected frequencylimited CALE (4.6) has no semidefinite solution, or no unique solution at all. Hence, the generated low-rank solution factors are not useful. We point out that a violation of the property $A+A^{T}<0$ does not at all mean that these problems have to occur inescapably in Krylov subspace methods for CALEs. In fact, we successfully ran several numerical tests, where $A+A^{T}$ was indefinite. If such issues really occur, 
the LR-ADI iteration discussed next might be an alternative as it is unaffected by indefinite $A+A^{T}$.

4.2.2. Using the LR-ADI iteration. The LR-ADI iteration is another well established, understood, and successfully applied method to solve large-scale matrix equations for low-rank solution factors. For the CALEs of the infinite Gramians, the LR-ADI iteration [39] in the form given in [5, 6] produces a low-rank solution factor of (2.6a) via $Z_{P, j}=\left[Z_{P, j-1}, \hat{\alpha}_{j} V_{j}\right]$, where

$$
V_{j}=\left(A+\alpha_{j} I_{n}\right)^{-1} W_{j-1}, \quad W_{j}=W_{j-1}-2 \operatorname{Re}\left(\alpha_{j}\right) V_{j},
$$

with $\hat{\alpha}_{j}:=\sqrt{-2 \operatorname{Re}\left(\alpha_{j}\right)}, W_{0}:=B, Z_{P, 0}:=[]$, and $\alpha_{j}, j=1, \ldots$, are shift parameters that are crucial for a fast convergence. Here, we employ a strategy proposed in [6], which produces the shifts adaptively in the course of the iteration. The original LRADI iteration, however, expects that the inhomogeneity of the CALE to be solved is given in a symmetric definite form, $B B^{T}$. However, the inhomogeneities of the frequency-limited CALEs (4.5) are given by

$$
\begin{aligned}
& B_{\Omega} B^{T}+B B_{\Omega}^{T}=\hat{B} F_{m} \hat{B}^{T}, \quad \hat{B}=\left[B, B_{\Omega}\right], \\
& C_{\Omega}^{T} C+C^{T} C_{\Omega}=\hat{C}^{T} F_{p} \hat{C}, \quad \hat{C}=\left[\begin{array}{c}
{ }_{C} \\
C_{\Omega}
\end{array}\right],
\end{aligned}
$$

with the flipping matrix $F_{h}$ from (3.8). Since $\lambda\left(F_{h}\right)=\{ \pm 1\}$, these inhomogeneities are in general indefinite matrices. To tackle the indefiniteness of the inhomogeneities, the $L D L^{T}$-variant [37, Algorithm 1], [38] of the LR-ADI iteration can be used. This will only slightly alter the iteration (4.8) and the computed approximate solution after $j$ iteration steps is of the form

$$
P_{\Omega} \approx P_{\Omega, j}^{\mathrm{ADI}}=Z_{P_{\Omega}, j}\left(I_{j} \otimes F_{m}\right) Z_{P_{\Omega}, j}^{T} .
$$

Although $I_{j} \otimes F_{m}$ is an indefinite matrix, we assume $P_{\Omega, j}^{\mathrm{ADI}} \succeq 0$ since $P_{\Omega} \succ 0$. In practice this might hold only if $P_{\Omega, j}^{\mathrm{ADI}}$ is a sufficiently accurate approximation of $P_{\Omega}$. A semidefinite factorization of $P_{\Omega, j}^{\mathrm{ADI}}$ can be obtained as follows: compute a thin QR-decomposition $U_{1} R=Z_{P_{\Omega}, j}$ followed by a spectral decomposition $U_{2} \hat{\Lambda} U_{2}^{T}=$ $R\left(I_{j} \otimes F_{m}\right) R^{T}$ with $U_{2}^{T} U_{2}=I_{2 m j}, \hat{\Lambda}=\operatorname{diag}\left(\hat{\lambda}_{1}, \ldots, \hat{\lambda}_{2 m j}\right)$. Then, the approximate solution $P_{\Omega, j}^{\mathrm{ADI}}$ can be represented by a semidefinite factorization

$$
P_{\Omega, j}^{\mathrm{ADI}}=\hat{Z}_{P_{\Omega}, j} \hat{Z}_{P_{\Omega}, j}^{T}, \quad \hat{Z}_{P_{\Omega}, j}:=U_{1} U_{2} \hat{\Lambda}^{\frac{1}{2}} .
$$

By neglecting very small eigenvalues $\hat{\lambda}$ and the corresponding columns of $U_{2}$, this procedure also enables a rank truncation of the approximate solution $P_{\Omega, j}^{\mathrm{ADI}}$, similar to line 10 in Algorithm 4.1, to get rid of nearly linearly dependent columns.

Alternatively, by observing that $P_{\Omega}=N_{\Omega}+N_{\Omega}^{T}$, where $N_{\Omega}$ solves the Sylvester equation

$$
A N_{\Omega}+N_{\Omega} A^{T}+B B_{\Omega}^{T}=0
$$

the modification of the factored ADI iteration [7], [4, Algorithm 4] can be applied directly to (4.9) and yields, after $j$ iteration steps,

$$
N_{\Omega} \approx N_{\Omega, j}=\tilde{Z}_{P_{\Omega}, j} \tilde{Y}_{P_{\Omega}, j}^{T}, \quad \tilde{Z}_{P_{\Omega}, j}, \tilde{Y}_{P_{\Omega}, j} \in \mathbb{R}^{n \times m j} .
$$


Hence, one obtains a low-rank approximation $P_{\Omega} \approx\left[\tilde{Z}_{P_{\Omega}, j}, \tilde{Y}_{P_{\Omega}, j}\right] F_{j m}\left[\tilde{Z}_{P_{\Omega}, j}, \quad \tilde{Y}_{P_{\Omega}, j}\right]^{T}$ which can be transformed into a semidefinite factorization using similar steps as above. Neglecting this transformation, both approaches are equivalent. The numerical effort of both methods is also identical and we use the $L D L^{T}$ version of the LR-ADI iteration in the remainder.

The additionally needed QR-decomposition for constructing the semidefinite lowrank factorization introduces additional costs. This can be seen as a disadvantage of the LR-ADI iteration. Another shortcoming is that the information from computing $B_{\Omega}$ is not reused which, as observed in practice, often leads to more required iteration steps of the LR-ADI iteration compared to the projection type approach mentioned above, e.g., Algorithm 4.1.

\section{Related problems.}

5.1. Generalized state-space systems. Until now we have considered only standard state-space systems, but everything can easily be modified to handle generalized state-space systems

$$
\begin{aligned}
E \dot{x}(t) & =A x(t)+B u(t), \\
y(t) & =C x(t)
\end{aligned}
$$

with a nonsingular $E \in \mathbb{R}^{n \times n}$ by using similar techniques as in the unlimited BT framework. As, e.g., shown in [50], the reachability and observability Gramians of (5.1) are $P, E^{T} Q E$, where $P, Q$ are the solutions of the GCALEs

$$
A P E^{T}+E P A^{T}=-B B^{T}, \quad A^{T} Q E+E^{T} Q A=-C^{T} C .
$$

Corollary 5.1 (frequency-limited Gramians for generalized systems). For a generalized state-space system (5.1) and the frequency intervals $\Omega$ in (2.3), the frequency-limited Gramians are $P_{\Omega}$ and $E^{T} Q_{\Omega} E$, which are obtained from either of the following two approaches:

1. Using the solutions $P$ and $Q$ of the ordinary reachability and observability and GCALEs (5.2), it holds that

$$
P_{\Omega}=F_{\Omega} E P+P E^{T} F_{\Omega}^{T}, \quad Q_{\Omega}=F_{\Omega}^{T} E^{T} Q+Q E F_{\Omega}
$$

with

$$
F_{\Omega}:=\frac{1}{2 \pi} \int_{\Omega}(\imath \nu E-A)^{-1} \mathrm{~d} \nu .
$$

2. The frequency-limited Gramians are given from the solutions of the frequencylimited reachability and observability GCALEs

$$
\begin{array}{ll}
A P_{\Omega} E^{T}+E P_{\Omega} A^{T}+B_{\Omega} B^{T}+B B_{\Omega}^{T}=0, & B_{\Omega}:=E F_{\Omega} B, \\
A^{T} Q_{\Omega} E+E^{T} Q_{\Omega} A+C_{\Omega}^{T} C+C^{T} C_{\Omega}=0, & C_{\Omega}:=C F_{\Omega} E .
\end{array}
$$

The matrix-valued integral can be represented in terms of the matrix logarithm via

$$
\begin{aligned}
F_{\Omega} & =\operatorname{Re}\left(\frac{\imath}{\pi} \ln \left(\left(A+\imath \omega_{1} E\right)^{-1}\left(A+\imath \omega_{2} E\right)\right)\right) E^{-1} \\
& =E^{-1} \operatorname{Re}\left(\frac{\imath}{\pi} \ln \left(\left(A+\imath \omega_{2} E\right)\left(A+\imath \omega_{1} E\right)^{-1}\right)\right) .
\end{aligned}
$$

Copyright $@$ by SIAM. Unauthorized reproduction of this article is prohibited. 
Proof. Using the equivalent standard state-space system defined by $\hat{A}:=E^{-1} A$, $\hat{B}:=E^{-1} B, C$ leads, by employing (2.5) and (5.4), to

$$
\frac{1}{2 \pi} \int_{\Omega}\left(\imath \nu I_{n}-\hat{A}\right)^{-1} \mathrm{~d} \nu=F_{\Omega} E
$$

which, by applying [46, Theorems 3.1-3.2] and (2.4), immediately gives (5.3). The reachability CALE w.r.t. $\hat{A}, \hat{B}$ is

$$
\begin{aligned}
0 & =\hat{A} P_{\Omega}+P_{\Omega} \hat{A}^{T}+F_{\Omega} E \hat{B} \hat{B}^{T}+\hat{B} \hat{B}^{T} F_{\Omega}^{T} E^{T} \\
& =E^{-1} A P_{\Omega}+P_{\Omega} A^{T} E^{-T}+F_{\Omega} B B^{T} E^{-T}+E^{-1} B B^{T} F_{\Omega}^{T} \\
\Leftrightarrow \quad 0 & =A P_{\Omega} E^{T}+E P_{\Omega} A^{T}+E F_{\Omega} B B^{T}+B B^{T} F_{\Omega}^{T} E^{T}
\end{aligned}
$$

and $(5.5 \mathrm{a})$ is established. The frequency-limited observability GCALE (5.5b) is derived using similar steps. For (5.6), first note that $F_{\Omega}=\frac{1}{2 \pi} \int_{\Omega}\left(\imath \nu I_{n}-\hat{A}\right)^{-1} \mathrm{~d} \nu E^{-1}$ from which (5.6a) easily follows by using Theorem 2.3. Alternatively, it holds that $F_{\Omega}=E^{-1} \frac{1}{2 \pi} \int_{\Omega}\left(\imath \nu I_{n}-A E^{-1}\right)^{-1} \mathrm{~d} \nu$, which again, by applying Theorem 2.3 , leads to $(5.6 \mathrm{~b})$.

The algorithms we suggested in the standard state-space case for computing approximations of $B_{\Omega}, C_{\Omega}, P_{\Omega}$, and $Q_{\Omega}$ are also applicable here with minor modifications. Some care must be taken when Krylov subspace methods are used for this purpose, since they implicitly work on $E^{-1} A$ or on $L^{-1} A L^{-T}$ if $0 \prec E=L L^{T}$. Hence, the correct formulation of $F_{\Omega}$ should be chosen. As an alternative, the use of the generalized LR-ADI (G-LR-ADI) iteration [6] for the GCALEs is straightforward.

5.2. Stability preservation and modified FLBT. The inhomogeneities of the Lyapunov equations (1.2) are in general indefinite and, hence, it is not guaranteed that stability is preserved in the reduced order model [19]. In [24], a modification of frequency-limited BT is presented that does preserve stability. Consider the eigenvalue decompositions of the inhomogeneities of (4.5) and (5.5):

$$
\begin{array}{ll}
B_{\Omega} B^{T}+B B_{\Omega}^{T}=Q_{B} S_{B} Q_{B}^{T}, & S_{B}=\operatorname{diag}\left(\eta_{1}^{B}, \ldots, \eta_{2 m}^{B}, 0, \ldots, 0\right) \in \mathbb{R}^{n \times n}, \\
C_{\Omega}^{T} C+C^{T} C_{\Omega}=Q_{C} S_{C} Q_{C}^{T}, & S_{C}=\operatorname{diag}\left(\eta_{1}^{C}, \ldots, \eta_{2 p}^{C}, 0, \ldots, 0\right) \in \mathbb{R}^{n \times n}
\end{array}
$$

with $Q_{B}^{T} Q_{B}=Q_{C}^{T} Q_{C}=I, Q_{B}, Q_{C} \in \mathbb{R}^{n \times n}$. Assuming that $\operatorname{rank}\left[B, B_{\Omega}\right]=r_{B} \leq$ $2 m$ and $\operatorname{rank}\left[C^{T}, C_{\Omega}^{T}\right]=r_{C} \leq 2 p$, it holds that $\eta_{i}^{B}, \eta_{j}^{C} \neq 0$ for $i=1, \ldots, r_{B}$, $j=1, \ldots, r_{C}$. However, there can be both negative and positive values of $\eta_{i}^{B}, \eta_{j}^{C}$. Now let $Q_{B, 1} \in \mathbb{R}^{n \times r_{B}}, Q_{C, 1} \in \mathbb{R}^{n \times r_{C}}$ be the first $r_{B}$ as well as $r_{C}$ columns of $Q_{B}, Q_{C}$, and consider the modified frequency-limited GCALEs

$$
\begin{aligned}
A P_{\Omega}^{\bmod } E^{T}+E P_{\Omega}^{\bmod } A^{T}+B_{\Omega}^{\bmod } B_{\Omega}^{\bmod T} & =0, \\
A^{T} Q_{\Omega}^{\bmod } E+E^{T} Q_{\Omega}^{\bmod } A+C_{\Omega}^{\bmod T} C_{\Omega}^{\bmod } & =0 \\
\text { with } \quad B_{\Omega}^{\bmod } & :=Q_{B, 1} \operatorname{diag}\left(\left|\eta_{1}^{B}\right|, \ldots,\left|\eta_{r_{B}}^{B}\right|\right)^{\frac{1}{2}}, \quad C_{\Omega}^{\bmod }:=\operatorname{diag}\left(\left|\eta_{1}^{C}\right|, \ldots,\left|\eta_{r_{C}}^{C}\right|\right)^{\frac{1}{2}} Q_{C, 1}^{T} .
\end{aligned}
$$

That is, the negative values in $S_{B}$ and $S_{C}$ are essentially simply negated. Notice that computing the $r_{B}, r_{C} \ll n$ nonzero eigenvalues $\eta_{i}^{B}, \eta_{j}^{C}$ and their corresponding eigenvectors can be done very inexpensively. Performing the balancing and truncation on the basis of these modified frequency-limited Gramians $P_{\Omega}^{\bmod }, Q_{\Omega}^{\bmod }$ yields modified FLBT (FLBT $\left.{ }^{\text {mod }}\right)$. This approach ensures that, under some mild conditions [24, 
Theorem 11], the reduced order model is asymptotically stable and that, similar to (1.4), the error bound

$$
\begin{gathered}
\|H-\tilde{H}\|_{\mathcal{H}_{\infty}} \leq 2\left\|J_{B}\right\|\left\|J_{C}\right\| \sum_{j=r+1}^{n} \sigma_{j}^{\bmod } \\
J_{B}:=\operatorname{diag}\left(\left|\eta_{1}^{B}\right|, \ldots,\left|\eta_{r_{B}}^{B}\right|\right)^{-\frac{1}{2}} Q_{B, 1}^{T} B, \quad J_{C}:=C Q_{C, 1} \operatorname{diag}\left(\left|\eta_{1}^{C}\right|, \ldots,\left|\eta_{r_{C}}^{C}\right|\right)^{-\frac{1}{2}}
\end{gathered}
$$

can be established, where the $\sigma_{j}^{\text {mod }}$ denotes the modified frequency-limited singular values, i.e., the square roots of the eigenvalues of $P_{\Omega}^{\bmod } Q_{\Omega}^{\bmod }$. The computation of low-rank factors of $P_{\Omega}^{\bmod }, Q_{\Omega}^{\bmod }$ can be carried out similarly as without this modification using Algorithm 4.1, i.e., after an accurate approximation of, e.g., $B_{\Omega}$ has been computed, $B_{\Omega}^{\bmod }$ is constructed as above. For reusing the generated Krylov basis to subsequently compute low-rank solution factors of $P_{\Omega}^{\bmod }$, some small changes are necessary. Since $B_{\Omega}^{\mathrm{mod}}$ is obtained by altering $B, B_{\Omega}$, the inhomogeneity of the projected, modified, frequency-limited GCALE cannot be built similar to (4.7). Hence, $B_{\Omega}^{\text {mod }}$ has to be projected explicitly via $Q_{k}^{T} B_{\Omega}^{\bmod }$. The inhomogeneities of (5.7) are symmetric positive semidefinite, such that the (G-)LR-ADI iteration can be applied directly. However, because $P_{\Omega}^{\bmod }, Q_{\Omega}^{\bmod }$ do not fulfill the relations (2.4) and (5.3), one cannot expect that they also exhibit a fast eigenvalue decay similar to $P_{\Omega}, Q_{\Omega}$. Some numerical experiments in section 6 show that the eigenvalues of $P_{\Omega}^{\bmod }, Q_{\Omega}^{\bmod }$ can even decay at a similar speed as those of the infinite Gramians $P, Q$. This can also lead to more iteration steps required by the applied Krylov subspace method or the G-LR-ADI iteration compared to $P_{\Omega}, Q_{\Omega}$.

5.3. Time-limited variants of BT. In [19], a series of related approaches is proposed which restrict BT for (1.1) in certain ways. One possibility is to consider a time interval $\mathbb{T}$, e.g., $\mathbb{T}=\left[0, t_{1}\right], t_{1}<\infty$. Restricting BT to $\mathbb{T}$ leads to time-limited $\mathrm{BT}$ and aims at finding a reduced model whose output $\tilde{y}$ is an accurate approximation of the original output $y$, but only within the time frame $\mathbb{T}$. This leads to time-limited Gramians $P_{\mathbb{T}}, Q_{\mathbb{T}}$ which are the solutions of the time-limited CALEs

$$
\begin{aligned}
A P_{\mathbb{T}}+P_{\mathbb{T}} A^{T}+B B^{T}-B_{\mathbb{T}} B_{\mathbb{T}}^{T} & =0, \\
A^{T} Q_{\mathbb{T}}+Q_{\mathbb{T}} A+C^{T} C-C_{\mathbb{T}}^{T} C_{\mathbb{T}} & =0, \\
\quad \text { where } \quad B_{\mathbb{T}} & :=F_{\mathbb{T}} B, C_{\mathbb{T}}:=C F_{\mathbb{T}}
\end{aligned}
$$

with $F_{\mathbb{T}}:=\mathrm{e}^{A t_{1}}$. Hence, the numerical approaches for FLBT presented above can be easily adjusted to this setting. The main difference is that one has to deal with the matrix-valued exponential. It is also possible to combine frequency- and time-limited $\mathrm{BT}$, where products of the form $F_{\Omega} F_{\mathbb{T}}$ occur.

5.4. Restricted BT for discrete-time systems. Both frequency- and timelimited BT can also be carried out for discrete-time systems

$$
x_{i+1}=A x_{i}+B u_{i}, \quad y_{i}=C x_{i} .
$$

Let $\Omega_{d}=\left[-\omega_{1}, \omega_{1}\right], \omega_{1}<\pi$, and $\mathbb{T}_{d}=\left[0, i_{1}\right], i_{1} \in \mathbb{N}_{+}$, be the considered frequency- and discrete-time intervals. The infinite, frequency-limited, and timelimited Gramians are the solutions of the discrete-time, algebraic Lyapunov equations (DALEs)

$$
\begin{aligned}
P-A P A^{T} & =B B^{T}, & Q-A^{T} Q A & =C^{T} C, \\
P_{\Omega_{d}}-A P_{\Omega_{d}} A^{T} & =B \Omega_{\Omega_{d}} B^{T}+B B_{\Omega_{d}}^{T}, & Q_{\Omega_{d}}-A^{T} Q_{\Omega_{d}} A & =C_{\Omega_{d}}^{T} C+C^{T} C_{\Omega_{d}}, \\
P_{\mathbb{T}_{d}}-A P_{\mathbb{T}_{d}} A^{T} & =B B^{T}+B_{\mathbb{T}_{d}} B_{\mathbb{T}_{d}}^{T}, & Q_{\mathbb{T}_{d}}-A^{T} Q_{\mathbb{T}_{d}} A & =C^{T} C+C_{\mathbb{T}_{d}}^{T} C_{\mathbb{T}_{d}},
\end{aligned}
$$

Copyright $@$ by SIAM. Unauthorized reproduction of this article is prohibited. 
where

$$
\begin{aligned}
& B_{\Omega_{d}}:=F_{\Omega_{d}} B, \quad C_{\Omega_{d}}:=C F_{\Omega_{d}}, \quad F_{\Omega_{d}}:=\frac{1}{2 \pi} \operatorname{Re}\left(\omega_{1} I-2 \imath \ln \left(I-A \mathrm{e}^{-\imath \omega_{1}}\right)\right), \\
& B_{\mathbb{T}_{d}}:=F_{\mathbb{T}_{d}} B, \quad C_{\mathbb{T}_{d}}:=C F_{\mathbb{T}_{d}}, \quad F_{\mathbb{T}_{d}}:=A^{i_{1}},
\end{aligned}
$$

see, e.g., $[19,46]$. Approximating the products with the different matrix-valued functions $F_{\Omega_{d}}, F_{\mathbb{T}_{d}}$ can be done exactly as we described above. Subsequently, computing low-rank solution factors of the DALEs can also be done by similar methods as we used before, which again enables an efficient realization of these BT variants also for large-scale systems. We plan to investigate the eigenvalue decay of the Gramians mentioned in this section, as well as specially tailored numerical algorithms for their approximation, in future work.

5.5. BT at a single frequency. Another conceptually very different modification is presented in [14], which tries to restrict BT to a single frequency $\omega_{1} \in \mathbb{R}_{+}$. There, the CALEs to be solved are

$$
A_{\omega_{1}} P_{\omega_{1}}+P_{\omega_{1}} A_{\omega_{1}}^{H}+B_{\omega_{1}} B_{\omega_{1}}^{H}=0, \quad A_{\omega_{1}}^{H} Q_{\omega_{1}}+Q_{\omega_{1}} A_{\omega_{1}}+C_{\omega_{1}}^{H} C_{\omega_{1}}=0
$$

where $B_{\omega_{1}}:=F_{\omega_{1}} B, C_{\omega_{1}}:=C F_{\omega_{1}}$ with $F_{\omega_{1}}:=\epsilon\left(\epsilon I_{n}+\imath \omega_{1} I_{n}-A\right)^{-1}$ and $A_{\omega_{1}}:=$ $\imath \omega_{1} I_{n}+F_{\omega_{1}}\left(\imath \omega_{1} I-A\right), \epsilon>0$. The CALEs are defined by complex data such that $P_{\omega_{1}}$, $Q_{\omega_{1}}$ will be complex. An extension of this approach to frequency intervals is under current research. First experiments in [14] raise the expectation that the eigenvalues of $P_{\omega_{1}}, Q_{\omega_{1}}$ also decay faster than those of $P, Q$.

6. Numerical examples. Here, we numerically evaluate the results of section 3 regarding the eigenvalue decay of the frequency-limited Gramians, the numerical approaches presented in section 4 to approximate the product with the matrix-valued function as well as the low-rank approximations of the GCALE solutions, and the accuracy of the reduced order models obtained by the considered BT variants employing these low-rank approximations. The numerical experiments are carried out in MATLAB 8.0.0.783 (R2012b) on an Intel Xeon CPU X5650 @ $2.67 \mathrm{GHz}$ with 48 GB RAM.

6.1. Eigenvalue decay and numerical rank of the Gramians. For illustrating the eigenvalue decay, we use a standard five-point, centered finite difference discretization of the differential equation

$$
\Delta v+10^{2} \xi_{1} \frac{\partial v}{\partial \xi_{1}}+10^{3} \xi_{2} \frac{\partial v}{\partial \xi_{2}}=0
$$

for $v=v\left(\xi_{1}, \xi_{2}\right)$ defined on $\Omega=(0,1)^{2}$ with homogeneous Dirichlet boundary conditions. Using $n_{0}=30$ equidistant grid points for each spatial dimension yields $n=n_{0}^{2}=900$ for $A$. The input matrix $B \in \mathbb{R}^{n}$ is chosen as vector with random entries from a normal distribution (initialized via randn('state', 0);). This small system admits an exact numerical computation of the controllability Gramians $P, P_{\Omega}$, and $P_{\Omega}^{\bmod }$ (cf. (5.7)) by using the lyap routine of MATLAB ${ }^{\circledR}$.

At first, the frequency interval boundaries are $\omega_{1}=10^{3}, \omega_{2}=10^{4}$ and the matrix valued function $F_{\Omega}$ is dealt with by the logm routine. The eigenvalues of all three Gramians $P, P_{\Omega}$, and $P_{\Omega}^{\bmod }$, scaled by their respective largest entry, are plotted in the left plot of Figure 1. Apparently, the eigenvalues of $P_{\Omega}$ decay significantly faster than the ones of $P$ and $P_{\Omega}^{\text {mod }}$, which decay nearly identically. From now on, we 

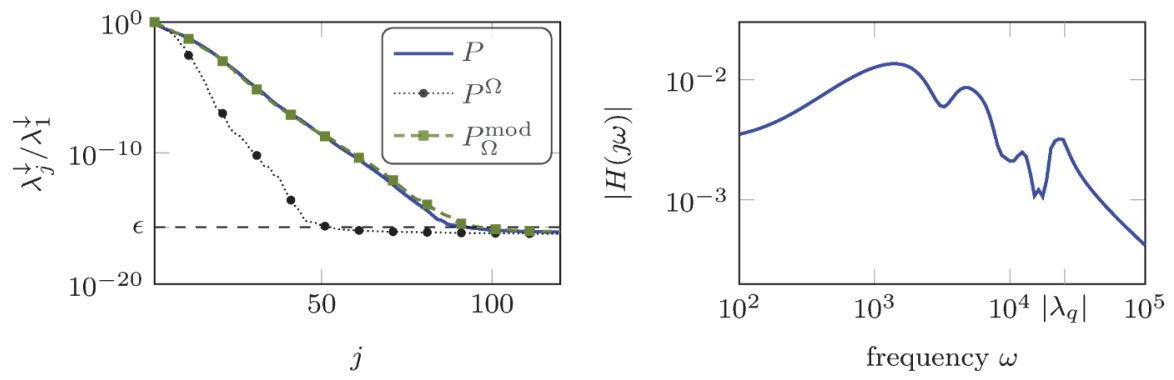

FIG. 1. Left: Scaled eigenvalues of the Gramians. Right: Transfer function plot of the system.

TABLE 1

Computed numerical rank of the different Gramians w.r.t. various frequency interval boundaries. Here, $\operatorname{rank}(P, \epsilon)=72$ and $\left|\lambda_{q}\right|=2.5337 \cdot 10^{4}$.

\begin{tabular}{|l|l|r|r|}
\hline$\omega_{1}, \omega_{2}$ & $\rho(\Gamma)$ & $\operatorname{rank}\left(P_{\Omega}, \epsilon\right)$ & $\operatorname{rank}\left(P_{\Omega}^{\bmod }, \epsilon\right)$ \\
\hline $10^{3}, 10^{4}$ & 0.43 & 39 & 74 \\
$10^{2}, 10^{3}$ & 0.21 & 10 & 69 \\
$10^{3},\left|\lambda_{q}\right|$ & 0.57 & 67 & 74 \\
$0,10^{5}$ & 0.49 & 74 & 74 \\
\hline
\end{tabular}

use the following definition of the numerical rank of a matrix $X$ w.r.t. a threshold $\tau \geq 0: \operatorname{rank}(X, \tau):=\operatorname{argmax}_{j}\left\{\sigma_{j}(X) / \sigma_{1}(X)>\tau\right\}$. The numerical rank of the infinite Gramian $P$ w.r.t. the machine precision $\epsilon=2.2204 \cdot 10^{-16}$ is $\operatorname{rank}(P, \epsilon)=72$.

In Table 1 we list the numerical ranks of the frequency-limited Gramian $P_{\Omega}$, of the modification $P_{\Omega}^{\bmod }$, and the spectral radius of the matrix $\Gamma$. For the used frequency interval, the numerical rank of $P_{\Omega}$ is, as predicted by Figure 1, noticeable smaller than the numerical rank of $P$. We also tested other frequency intervals, where we used the quantity $\left|\lambda_{q}\right|$ from Lemma 3.3 , i.e., the magnitude of the eigenvalue at which $\frac{|\operatorname{Im}(\lambda)|}{|\operatorname{Re}(\lambda)|}$ is maximal. Notice that in the right plot of Figure 1, the Bode magnitude plot of the transfer function matrix $H$ shows a distinct bulk near $\left|\lambda_{q}\right|$. According to the discussion in the end of section 3 , setting the interval boundaries equal to $\left|\lambda_{q}\right|$ can increase the spectral radius $\rho(\Gamma)$ and, thus, slow down the eigenvalue decay of $P_{\Omega}$. The results in the last row confirm this for the choice $\omega_{2}=\left|\lambda_{q}\right|$ which yields $\rho(\Gamma)>\frac{1}{2}$ and very close numerical ranks of $P_{\Omega}$ and $P$. In conclusion, the results in Table 1 seem to confirm the expected influence of $\rho(\Gamma)$ on $\operatorname{rank}\left(P_{\Omega}, \epsilon\right)$. For the last frequency interval $\left[0,10^{5}\right]$, one can also see that $\rho(\Gamma)$ approaches $\frac{1}{2}$ for increasingly large frequency intervals. The numerical rank of the modified frequency-limited Gramian $P_{\Omega}^{\bmod }$ appears to be largely unaffected by different frequency intervals and is always very close to the numerical rank of $P$.

6.2. Influence of the different inhomogeneities to the low-rank solvers. In the subsequent numerical experiments, we use the test systems summarized in Table 2, which also gives the frequency interval boundaries $\omega_{1}, \omega_{2}$ that are chosen purely for testing purposes and without any background from the applications. The example $f d m$ is actually obtained by using $n_{0}=350$ grid points in the construction of the small system above. The other test systems mainly represent finite element discretizations (w.r.t. the spacial dimensions) of similar partial differential equations.

Before we test the proposed Algorithm 4.1 and carry it out using the computed low-rank GCALE solutions, we consider how the different low-rank solvers perform 
TABLE 2

Dimensions, frequency interval boundaries, matrix properties, and source of the test systems. Here, $O C$ is the Oberwolfach Model Reduction Benchmark Collection and IFISS refers to the finite element package; see, e.g., [51].

\begin{tabular}{|l|c|c|c|c|l|l|}
\hline Example & $n$ & $m$ & $p$ & $\omega_{1}, \omega_{2}$ & Properties & Source \\
\hline chip & 20082 & 1 & 5 & $10,10^{2}$ & $E$ spd & OC $^{1}$, ID =38867 \\
\hline ifiss & 66049 & 5 & 5 & $1,10^{2}$ & $E$ spd, $B, C$ random, randn('state', 21314) & IFISS [51] T-CD3 \\
\hline rail & 79841 & 7 & 6 & $10^{-2}, 10$ & $A$ snd, $E$ spd & OC $^{1}$, ID=38881 \\
\hline fdm & 122500 & 5 & 5 & $10,10^{3}$ & $E=I, B, C$ random, randn('state', 0) & {$[44]$} \\
\hline
\end{tabular}

when applied to the standard GCALE (5.2), the frequency-limited GCALEs (2.4) and (5.3), as well as the modified frequency-limited GCALE (5.7). In other words, we investigate how the performance of the low-rank solvers differs w.r.t. to the different inhomogeneities, as these are the only differences in all three GCALEs. For this, we restrict the investigation to the controllability Gramians $P, P_{\Omega}$, and $P_{\Omega}^{\bmod }$. Since the direct calculation of the matrix-valued logarithm via the logm command is too memory and time consuming, we use approximations of $B_{\Omega}$ obtained by numerical quadrature employing the integral command. The necessary eigenvalues and eigenvectors to construct $B_{\Omega}^{\bmod }$ for (5.7) (cf. section 5.2) are computed using the eigs routine, which took less than one second in all cases. We employ the EKSM [52], the RKSM with the convex hull based adaptive shifts $(\operatorname{RKSM}(\mathcal{D}))[12]$, as well as the LR-ADI iteration $[44,5,6]$ (and its $\mathrm{LDL}^{T}$-variant $[38]$ for $P_{\Omega}$ ) for computing the low-rank solution factors. We point out that computing low-rank solution factors of $P_{\Omega}$ and $P_{\Omega}^{\bmod }$ in this way only serves a comparative purpose. As shown later in section 6.3, using the proposed approach in Algorithm 4.1 is clearly more practical and efficient as it also provides approximations to $B_{\Omega}$ at once. The shifts for the LR-ADI iteration are generated adaptively using the $V$-shift strategy proposed in [6]. Only for the symmetric rail example are the approximate Wachspress shifts [8] used because these led to the fastest convergence. All methods are terminated as before when the scaled GCALE residual norm (cf. line 8 in Algorithm 4.1) drops below $\tau_{P}=10^{-8}$. Afterward, a rank truncation (cf. lines 9-10 in Algorithm 4.1) is invoked, where all eigenvalues of the low-rank solution with $\lambda / \lambda_{\max } \leq 10^{-12}$ are neglected. The required subspace dimensions $d=\operatorname{dim}(\mathcal{Q})$, the ranks $g$ of the obtained low-rank solutions after this truncation, as well as the computation times $t_{c}$ for all methods and test systems are summarized in Table 3. Due to the applied rank truncation, $g$ also coincides with the column dimension of the low-rank solution factors. For the LR-ADI methods, $d$ is the column dimension of the computed low-rank solution factor.

The different inhomogeneities of the frequency-limited GCALEs (2.4) and (5.3) clearly affect the performance of EKSM and RKSM. As expected in section 3, the ranks $g$ of the low-rank approximations of $P_{\Omega}$ are smaller compared to the approximations of $P$. However, the required subspace dimensions $d$ for the low-rank approximations of $P_{\Omega}$ are smaller only for the examples $f d m$ and chip, where the computation time is also smaller compared to that for $P$. For the ifiss and rail examples, these differences are less pronounced since the subspace dimensions $d=\operatorname{dim}(\mathcal{Q})$ and computation times $t_{c}$ are higher for $P_{\Omega}$. However, recalling that the inhomogeneities of (2.4) and (5.3) are of rank $2 m$, one can see that the number of required iteration steps is actually less compared with $P$. For the rail system, the ranks of the approximations for $P$ and $P_{\Omega}$ show a less pronounced difference, which also explains the higher subspace dimensions. The LR-ADI iteration seems to be somewhat less affected by the

\footnotetext{
${ }^{1}$ Available at http://portal.uni-freiburg.de/imteksimulation/downloads/benchmark.
} 
TABLE 3

Required subspace dimension $d$, rank $g$, and computation time $t_{c}$ (in seconds) of the approximate low-rank solutions of $P, P_{\Omega}$, and $P_{\Omega}^{\text {mod }}$ by different methods.

\begin{tabular}{|l|l|r|r|r|r|r|r|r|r|r|}
\hline \multirow{2}{*}{ Example } & & \multicolumn{4}{|c|}{$P$} & \multicolumn{4}{|c|}{$P_{\Omega}$} & \multicolumn{3}{|c|}{$P_{\Omega}^{\text {mod }}$} \\
\cline { 2 - 10 } chip & Method & $d$ & $g$ & $t_{c}$ & $d$ & $g$ & $t_{c}$ & $d$ & $g$ & $t_{c}$ \\
& EKSM & 56 & 23 & 27.44 & 16 & 8 & 5.43 & 88 & 22 & 24.79 \\
& RKSM & 23 & 22 & 25.47 & 14 & 8 & 9.02 & 34 & 22 & 19.76 \\
& LR-ADI & 24 & 24 & 24.31 & 40 & 11 & 22.48 & 54 & 27 & 28.23 \\
\hline \multirow{3}{*}{ fiss } & EKSM & 890 & 340 & 319.07 & 940 & 235 & 236.12 & 1160 & 346 & 392.75 \\
& RKSM & 335 & 319 & 169.01 & 510 & 240 & 217.27 & 970 & 356 & 567.37 \\
& LR-ADI & 405 & 343 & 66.39 & 930 & 248 & 115.72 & 1160 & 376 & 142.81 \\
\hline \multirow{3}{*}{$f d m$} & EKSM & 812 & 202 & 157.38 & 1260 & 190 & 304.12 & 952 & 213 & 149.72 \\
& RKSM & 238 & 202 & 71.05 & 280 & 173 & 65.07 & 336 & 207 & 84.12 \\
& LR-ADI & 266 & 199 & 69.48 & 532 & 182 & 118.11 & 532 & 204 & 103.87 \\
\hline
\end{tabular}

different inhomogeneities since the computation times for $P_{\Omega}$ are in the majority of cases larger than for $P$. It achieves, however, the smallest computation times for the infinite Gramian $P$ for the examples fdm, ifiss, and chip. The time for the rail example is also comparable but slightly higher than the times of EKSM and RKSM. Note that we did not include further recent improvements of RKSM [13], which could accelerate its performance. An implementation of this method is not available to the authors. A basic reduction of the computational complexity of both EKSM and RKSM is to solve the reduced GCALE only every couple steps, e.g., every fifth iteration step. In most cases, computing the low-rank solution factors of the modified frequency-limited Gramians $P_{\Omega}^{\bmod }$ seems to be more demanding for all methods compared to $P, P_{\Omega}$. Apparently, the ranks of the computed low-rank approximations for $P_{\Omega}^{\bmod }$ are very close to those for $P$, which is one explanation for the greater numerical effort.

6.3. Numerical approximations for $f(A) b$ and the Gramians. Here, we evaluate the numerical strategies presented in section 4.1 for obtaining approximations of $B_{\Omega}$ and $P_{\Omega}$. We employ the projection approach given in Algorithm 4.1 for different choices of Krylov subspaces. The results are summarized in Table 4. For RKSM, the abbreviations $(\mathcal{D}),(\imath \Omega),\left(\imath\left[\omega_{1}, \omega_{2}\right]\right)$ refer to the use of the adaptive shifts based on the convex hull, the imaginary interval $\imath \Omega$, and the alternating usage of $\imath \omega_{1}, \imath \omega_{2}$, and their complex conjugates. The approximation of $B_{\Omega}$ is regarded as accurate enough when the criterion (4.4) is satisfied with $\tau_{f}=10^{-8}$. After reaching this condition, Algorithm 4.1 continues with computing the low-rank solution factor of $P_{\Omega}$, until the criterion based on the Lyapunov residual norm in line 8 with $\tau_{P}=10^{-8}$ is satisfied. In case of $\operatorname{RKSM}(\imath \Omega)$ and $\operatorname{RKSM}\left(\imath\left[\omega_{1}, \omega_{2}\right]\right)$, these iteration steps devoted to the GCALE solution were carried out using the convex hull based adaptive shifts, i.e., $\operatorname{RKSM}(\mathcal{D})$. We also list the dimension $d=\operatorname{dim}(\mathcal{Q})$ of the generated subspaces. More precisely, $d=d_{B_{\Omega}}+d_{P_{\Omega}}$, where $d_{B_{\Omega}}$ denotes the subspace dimension necessary to approximate $B_{\Omega}$ to the desired accuracy and $d_{P_{\Omega}}$ denotes the number of (additional) basis vectors required to achieve also the desired accuracy w.r.t. the GCALEs. The quadrature approximation of $B_{\Omega}$ from the examples in section 6.2 serves as reference solution

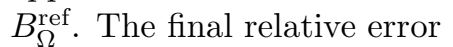

$$
\mathcal{R}_{\text {final }}:=\left\|B_{\Omega}^{\mathrm{ref}}-\tilde{B}_{\Omega}\right\| /\left\|B_{\Omega}^{\mathrm{ref}}\right\|,
$$


TABLE 4

Results for approximating $B_{\Omega}, P_{\Omega}$ by Algorithm 4.1 using extended and rational Krylov subspaces with different (adaptive) shifts.

\begin{tabular}{|c|c|c|c|c|c|c|c|}
\hline \multirow{2}{*}{\begin{tabular}{|l|} 
Example \\
\end{tabular}} & Method & \multicolumn{3}{|c|}{$d d_{B_{\Omega}} d_{P_{\Omega}}$} & $\overline{\mathcal{R}_{\text {final }}}$ & $\overline{\mathcal{L}_{\text {final }}}$ & $t_{\mathrm{c}}$ \\
\hline & EKSM & & 26 & 0 & $2.12 \cdot 10^{-9}$ & $6.36 \cdot 10^{-9}$ & 14.23 \\
\hline \multirow{3}{*}{$\begin{array}{l}\text { chip, } \\
t_{\text {quad }}=279\end{array}$} & $\operatorname{RKSM}(\mathcal{D})$ & 31 & 29 & 2 & $2.64 \cdot 10^{-9}$ & $1.56 \cdot 10^{-9}$ & 32.56 \\
\hline & $\operatorname{RKSM}(\imath \Omega)$ & 14 & 14 & 0 & $6.84 \cdot 10^{-11}$ & $1.98 \cdot 10^{-10}$ & 14.16 \\
\hline & $\operatorname{RKSM}\left(\imath\left[\omega_{1}, \omega_{2}\right]\right)$ & 16 & 16 & 0 & $1.16 \cdot 10^{-12}$ & $2.20 \cdot 10^{-11}$ & 16.10 \\
\hline \multirow{2}{*}{\begin{tabular}{|l}
$i f i s s$, \\
$t_{\text {quad }}=788$ \\
\end{tabular}} & $\operatorname{RKSM}(\mathcal{D})$ & 435 & 435 & 0 & $5.78 \cdot 10^{-9}$ & $4.35 \cdot 10^{-9}$ & 273.51 \\
\hline & $\operatorname{RKSM}(\imath \Omega)$ & 290 & 290 & 0 & $2.87 \cdot 10^{-9}$ & $1.89 \cdot 10^{-9}$ & 153.00 \\
\hline \multirow{4}{*}{$\begin{array}{l}\text { rail, } \\
t_{\text {quad }}=556\end{array}$} & EKSM & 868 & 868 & $\overline{0}$ & $8.06 \cdot 10^{-9}$ & $7.79 \cdot 10^{-9}$ & 421.12 \\
\hline & $\operatorname{RKSM}(\mathcal{D})$ & 322 & 322 & & $1.76 \cdot 10^{-8}$ & $5.79 \cdot 10^{-9}$ & 131.73 \\
\hline & $\operatorname{RKSM}(\imath \Omega)$ & 280 & 280 & 0 & $1.93 \cdot 10^{-8}$ & $1.66 \cdot 10^{-8}$ & 74.38 \\
\hline & $\operatorname{RKSM}\left(\imath\left[\omega_{1}, \omega_{2}\right]\right)$ & 406 & 266 & 140 & $1.19 \cdot 10^{-8}$ & $6.12 \cdot 10^{-8}$ & 115.37 \\
\hline \multirow{4}{*}{$\begin{array}{l}f d m, \\
t_{\text {quad }}=374\end{array}$} & EKSM & 130 & 130 & 0 & $2.44 \cdot 10^{-10}$ & $2.26 \cdot 10^{-10}$ & 23.50 \\
\hline & $\operatorname{RKSM}(\mathcal{D})$ & 325 & 325 & 0 & $1.18 \cdot 10^{-8}$ & $1.08 \cdot 10^{-8}$ & 178.73 \\
\hline & $\operatorname{RKSM}(\imath \Omega)$ & 70 & 70 & 0 & $1.81 \cdot 10^{-11}$ & $1.64 \cdot 10^{-11}$ & 23.93 \\
\hline & $\operatorname{RKSM}\left(\imath\left[\omega_{1}, \omega_{2}\right]\right)$ & 70 & 70 & 0 & $1.16 \cdot 10^{-11}$ & $1.07 \cdot 10^{-11}$ & 24.06 \\
\hline
\end{tabular}

the final normalized GCALE residual norm $\mathcal{L}_{\text {final }}$ obtained after a rank truncation, as well as the consumed computing times $t_{\text {quad }}$ and $t_{\mathrm{c}}$ in seconds for the quadrature approximation $B_{\Omega}^{\text {ref }}$ and the Krylov method (Algorithm 4.1), respectively, are also given.

Apparently, the projection approaches need less time than the quadrature approximation. Also, once the approximation of $B_{\Omega}$ is found, in all but two situations, there are no additional basis vectors necessary for generating the low-rank solution factor of $P_{\Omega}$. Using RKSM with adaptively computed shifts based on $\imath \Omega$ appears to be the best choice regarding the required subspace dimensions (which directly reflect the required iteration steps) as well as the consumed computation time. $\operatorname{RKSM}(\mathcal{D})$ leads to higher subspace dimensions and longer computing times for all test systems. The simplification $\operatorname{RKSM}\left(\imath\left[\omega_{1}, \omega_{2}\right]\right)$ of $\operatorname{RKSM}(\imath \Omega)$ as well as EKSM are competitive candidates only for the $f d m$ and chip examples, whereas they required larger subspaces for the rail example and failed to compute accurate approximations for the ifiss example. Notice that in section 6.3, EKSM did not manage to compute a lowrank solution factor of $P_{\Omega}$ (cf. Table 3 ) for the ifiss system. To conclude, using the adaptive, purely imaginary shifts from $\imath \Omega$ leads to a fast convergence of RKSM for approximating $B_{\Omega}$ and sufficiently accurate low-rank solutions of the frequency-limited GCALEs are obtained immediately from the generated rational Krylov basis.

6.4. Reduction results. Now we carry out the standard BT, the frequencylimited BT, the and its stability preserving modification [25] on the basis of low-rank solutions of the respective GCALEs. For the infinite Gramians $P, Q$, we use the lowrank solution method that achieved the smallest time in the experiment in section 6.2 (cf. Table 3). The low-rank factors of $P_{\Omega}$ are selected from the fastest method from Table 4 . The observability Gramians as well as the modified frequency-limited Gramians $P_{\Omega}^{\text {mod }}, Q_{\Omega}^{\text {mod }}$ are similarly dealt with by Algorithm 4.1 with the same settings of $\tau_{f}, \tau_{P}$. The obtained low-rank solution factors are used within Algorithm 1.1 to carry out the three BT variants to generate reduced order models of a prescribed order $r$. It is noteworthy that no significantly differing reduced order models were constructed when another method was employed to compute the low-rank solution 
TABLE 5

Reduction result obtained by different BT versions.

\begin{tabular}{|c|c|c|c|c|c|c|}
\hline Example, settings & BT type & Algorithms & $t_{\mathrm{MOR}}$ & $\mathcal{E}^{\max }(\Omega)$ & Bound & Stable? \\
\hline \multirow{3}{*}{$\begin{array}{l}\text { chip } \\
{\left[\begin{array}{l}\left.\omega_{1}, \omega_{2}\right]=\left[10,10^{2}\right], \\
r=8\end{array}\right.}\end{array}$} & BT & LR-ADI & 59.49 & $1.39 \cdot 10^{-3}$ & $4.7 \cdot 10^{-2}$ & 1 \\
\hline & FLBT & $\operatorname{RKSM}(\imath \Omega)$ & 30.16 & $4.56 \cdot 10^{-7}$ & & 1 \\
\hline & FLBT $^{\text {mod }}$ & $\operatorname{RKSM}(\imath \Omega)$ & 63.12 & $6.32 \cdot 10^{-4}$ & $4.94 \cdot 10^{-1}$ & 1 \\
\hline \multirow{3}{*}{$\begin{array}{l}\text { ifiss } \\
{\left[\omega_{1}, \omega_{2}\right]=\left[1,10^{2}\right],} \\
r=160\end{array}$} & $\mathrm{BT}$ & LR-ADI & 147.89 & $1.48 \cdot 10^{-4}$ & $1.9 \cdot 10^{2}$ & 1 \\
\hline & FLBT & $\operatorname{RKSM}(\imath \Omega)$ & 276.70 & $8.82 \cdot 10^{-6}$ & & 1 \\
\hline & FLBT $^{\text {mod }}$ & $\operatorname{RKSM}(\imath \Omega)$ & 573.27 & $5.68 \cdot 10^{-5}$ & $1.2 \cdot 10^{3}$ & 1 \\
\hline \multirow{3}{*}{$\begin{array}{l}\text { rail } \\
{\left[\omega_{1}, \omega_{2}\right]=\left[10^{-2}, 10\right]} \\
r=50\end{array}$} & $\mathrm{BT}$ & $\operatorname{RKSM}(\mathcal{D})$ & 126.07 & $1.53 \cdot 10^{-2}$ & $1.54 \cdot 10^{-4}$ & 1 \\
\hline & FLBT & $\operatorname{RKSM}(\imath \Omega)$ & 150.92 & $2.59 \cdot 10^{-3}$ & & 1 \\
\hline & FLBT $^{\text {mod }}$ & $\operatorname{RKSM}(\imath \Omega)$ & 209.51 & $2.41 \cdot 10^{-2}$ & $3.42 \cdot 10^{-4}$ & 1 \\
\hline \multirow{3}{*}{$\begin{array}{l}f d m \\
{\left[\omega_{1}, \omega_{2}\right]=\left[\begin{array}{ll}10,10^{3}\end{array}\right],} \\
r=30\end{array}$} & BT & LR-ADI & 230.23 & $8.76 \cdot 10^{-2}$ & $1.1 \cdot 10^{-1}$ & 1 \\
\hline & FLBT & EKSM & 47.82 & $1.03 \cdot 10^{-7}$ & - & 0 \\
\hline & FLBT $^{\text {mod }}$ & $\operatorname{RKSM}(\imath \Omega)$ & 350.54 & $7.61 \cdot 10^{-2}$ & 2.17 & 1 \\
\hline
\end{tabular}

factors. The relative error of the obtained reduced order models is

$$
\mathcal{E}(\omega)=\|H(\imath \omega)-\tilde{H}(\imath \omega)\|_{2} /\|H(\imath \omega)\|_{2}, \quad \omega \in \mathbb{R}_{+},
$$

with the transfer function matrices $H$ and $\tilde{H}$ of the original and reduced systems as in (1.5). For each test system, the Bode magnitude plots of $H, \tilde{H}$ and the relative errors $\mathcal{E}(\omega)$ are shown in Figure 2, where the thick vertical lines indicate the frequency interval boundaries $\omega_{1}, \omega_{2}$. We also indicate the theoretical error bounds (1.4) and (5.8) of $\mathrm{BT}$ and $\mathrm{FLBT}^{\mathrm{mod}}$, respectively, computed on the basis of the obtained approximate singular values. For the ifiss example, (1.4) and (5.8) overestimate the true error by several orders of magnitude (cf. Table 5) and are thus not shown in the respective error plot in Figure 2. While the reduced systems from BT match $H$ in the entire frequency range, those obtained with the frequency-limited variants FLBT, FLBT ${ }^{\text {mod }}$ show slight deviations outside the considered frequency interval $\left[\omega_{1}, \omega_{2}\right]$. The relative error plots clarify this as they reveal smaller errors obtained by the frequency-limited approaches within $\left[\omega_{1}, \omega_{2}\right]$, where especially unmodified FLBT yields superior approximations. Except for example rail, FLBT also achieves a somewhat higher accuracy for $\omega \leq \omega_{1}$.

To quantitatively measure the approximation quality, we consider the largest relative error within the relevant frequency region $\Omega$ via

$$
\mathcal{E}^{\max }(\Omega):=\max _{\omega \in\left[\omega_{1}, \omega_{2}\right]} \mathcal{E}(\omega) .
$$

The results are given in Table 5 , which also includes the overall time $t_{\mathrm{MOR}}$, which sums up the computation time to acquire the low-rank Gramian factors and the generation of the reduced order model by Algorithms 4.1 and 1.1, respectively. We also indicate if the constructed reduced system is asymptotically stable.

It can be clearly confirmed that FLBT provides reduced order systems with the best approximation quality in $\Omega$ for all test systems. Hence, the goal, mentioned in the beginning, to achieve better accuracies at the same reduced order is fulfilled. For comparison, BT achieves a comparable accuracy in $\Omega$, e.g., for system ifiss, if the reduced order is increased to $r \approx 200$. Regarding the computation times $t_{\mathrm{MOR}}$, FLBT is in some cases more expensive due to the required handling of the matrix function. If an efficient numerical low-rank approach is used, computing the required low-rank solution factors of the frequency-limited Gramians can, however, be cheaper compared 

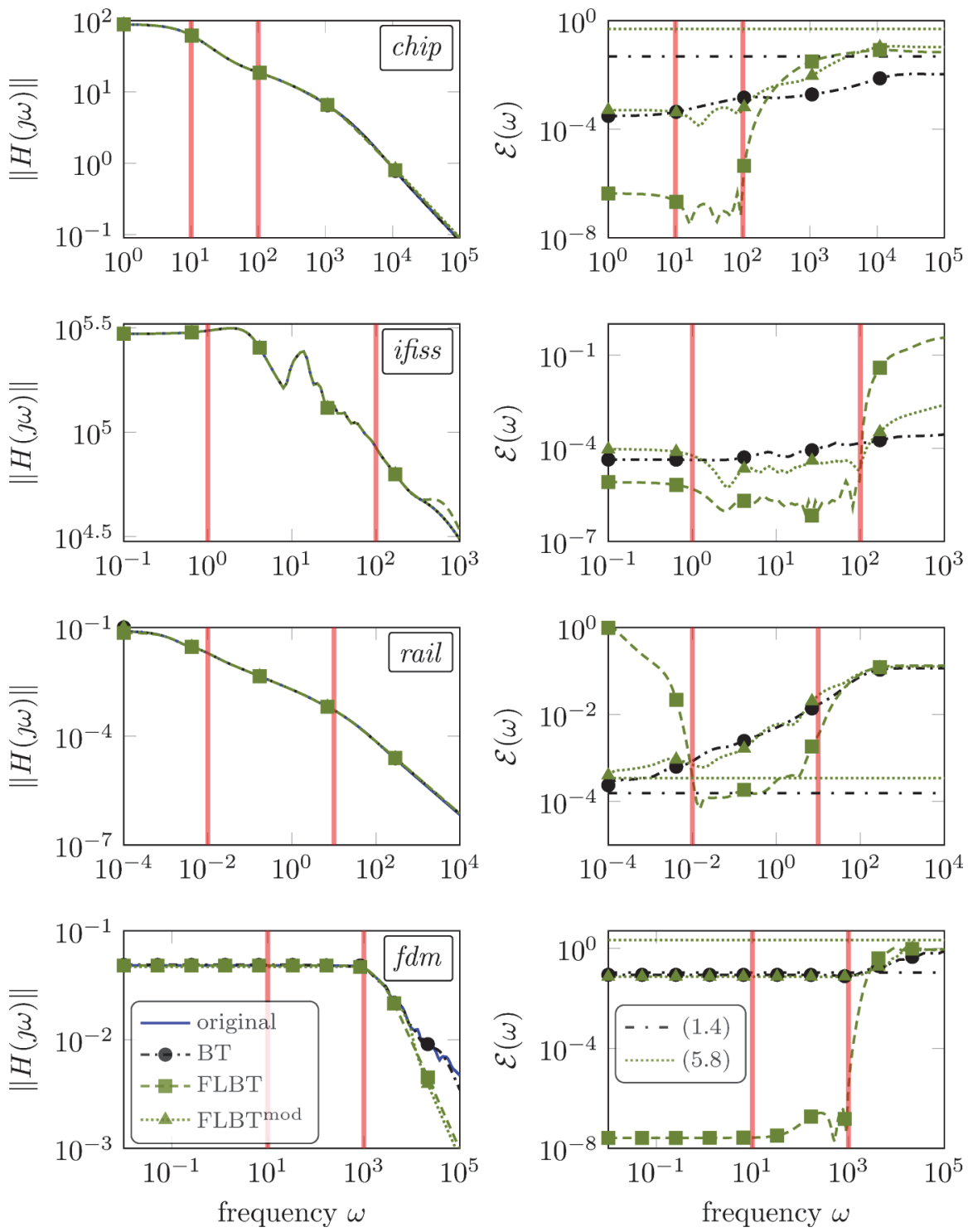

FIG. 2. Results obtained by different BT versions. Left: Bode magnitude plot of original and reduced transfer functions. Right: Relative errors and theoretical error bounds.

to the infinite Gramians. This is especially the case when the numerical ranks of the frequency-limited Gramians are noticeable smaller than those of the infinite ones. In our examples this is the case for the chip and fdm examples. There, carrying out FLBT requires less time than BT.

For the $f d m$ example, FLBT returned an asymptotically unstable reduced system. This was also observed for the other systems for some smaller reduced dimensions $r$. The stability preserving modification FLBT ${ }^{\text {mod }}$ does always provide a stable reduced order model, but the computation times are significantly higher than for BT and FLBT. Similar to the experiments in section 6.2 , it seems to be harder to solve (5.7) for low-rank solutions. Moreover, this stability preservation appears to sacrifice the 
obtained accuracy in $\left[\omega_{1}, \omega_{2}\right]$. In particular for the rail and fdm systems, the accuracy of $\mathrm{FLBT}^{\mathrm{mod}}$ is close to the accuracy of ordinary BT, which renders the introduction of the frequency limitations essentially redundant. If stability preservation is really crucial and FLBT fails on that score, but if also the efficient numerical realization of the model order reduction approach is of importance, we recommend to still use BT without frequency limitations.

It is also important to point out that, for the rail example, Figure 2 and Table 5 show a violation of both theoretical error bounds (1.4), (5.8) for BT, FLBT ${ }^{\text {mod }}$. For the example $f d m$ only the BT bound (1.4) is violated. This is most likely caused by the usage of approximate GCALE solutions since (1.4), (5.8) are proven only when exact solution factors are used.

7. Conclusion and outlook. We considered BT restricted to limited frequency intervals. Compared to standard BT, a matrix-valued function occurs now in the definitions of the Gramians which have to be computed. The effects of this matrix-valued function on the eigenvalues of the Gramians within frequency-limited BT have been investigated and the established bounds indicate that the frequency-limited Gramians have faster decaying eigenvalues than the infinite Gramians, which also results in a smaller numerical rank. Due to some of the employed eigenvalue inequalities, the found bounds are not very tight such that further research should be devoted in that direction. The matrix-valued functions, as well as the computation of low-rank solution factors of the occurring GCALEs, can be dealt with efficiently by a single RKSM employing suitable shift parameters. This proposed method was in the numerical test superior to other approaches, e.g., the EKSM or the LR-ADI iteration. We plan to introduce further improvements of RKSM $[13,40]$ for this purpose in the future. Carrying out FLBT using this method leads to reduced order models of better accuracy compared to BT. In some situations, the numerical effort of FLBT was even smaller because of the lower effort to approximate the frequency-limited Gramians. The stability preserving modified FLBT approach was also considered, but although similar techniques can be employed, the occurring modified, frequency-limited CALEs appear to be much harder to solve for low-rank solution factors, mainly regardless of the used method. Improving the handling of these modified Gramians, as well as related approaches with restrictions on time or for discrete-time systems, are also further research perspectives.

\section{REFERENCES}

[1] A. C. Antoulas, D. C. Sorensen, And Y. Zhou, On the decay rate of Hankel singular values and related issues, Systems Control Lett., 46 (2002), pp. 323-342.

[2] A. C.Antoulas, Approximation of Large-Scale Dynamical Systems, SIAM, Philadelphia, 2005.

[3] J. BAKer, M. Embree, And J. SABino, Fast singular value decay for Lyapunov solutions with nonnormal coefficients, SIAM J. Matrix Anal. Appl., 36 (2015), pp. 656-668.

[4] P. Benner And P. Kürschner, Computing real low-rank solutions of Sylvester equations by the factored ADI method, Comput. Math. Appl., 67 (2014), pp. 1656-1672.

[5] P. Benner, P. Kürschner, And J. SaAk, A reformulated low-rank ADI iteration with explicit residual factors, Proc. Appl. Math. Mech., 13 (2013), pp. 585-586.

[6] P. Benner, P. Kürschner, And J. SAak, Self-generating and efficient shift parameters in ADI methods for large Lyapunov and Sylvester equations, Electron Trans. Numer. Anal., 43 (2014), pp. 142-162.

[7] P. Benner, R.-C. Li, and N. Truhar, On the ADI method for Sylvester equations, J. Comput. Appl. Math., 233 (2009), pp. 1035-1045.

[8] P. Benner, H. Mena, And J. SaAk, On the parameter selection problem in the NewtonADI iteration for large-scale Riccati equations, Electron. Trans. Numer. Anal., 29 (2008), pp. 136-149.

Copyright (c) by SIAM. Unauthorized reproduction of this article is prohibited. 
[9] P. Benner And J. SAAK, Numerical solution of large and sparse continuous time algebraic matrix Riccati and Lyapunov equations: A state of the art survey, GAMM-Mitt., 36 (2013), pp. $32-52$.

[10] P. I. Davies and N. J. Higham, Computing $f(A) b$ for matrix functions $f$, in QCD and Numerical Analysis III, A. Boriçi, A. Frommer, B. Joó, A. Kennedy, and B. Pendleton, eds., Lect. Notes Comput. Sci. Eng. 47, 5 Springer, Berlin, 2005, pp. 15-24.

[11] V. Druskin and L. Knizhnerman, Extended Krylov subspaces: Approximation of the matrix square root and related functions, SIAM J. Matrix Anal. Appl., 19 (1998), pp. 755-771.

[12] V. Druskin and V. Simoncini, Adaptive rational Krylov subspaces for large-scale dynamical systems, Systems Control Lett., 60 (2011), pp. 546-560.

[13] V. Druskin, V. Simoncini, And M. Zaslavsky, Adaptive tangential interpolation in rational Krylov subspaces for MIMO dynamical systems, SIAM J. Matrix Anal. Appl., 35 (2014), pp. $476-498$.

[14] X. Du, P. Benner, G. YAng, And D. Ye, Balanced truncation of linear time-invariant systems at a single frequency, Preprint MPIMD/13-02, Max Planck Institute, Magdeburg, 2013; also available from http://www.mpi-magdeburg.mpg.de/preprints/.

[15] A. El Guennouni, K. Jbilou, And A. Riquet, Block Krylov subspace methods for solving large Sylvester equations, Numer. Algorithms, 29 (2002), pp. 75-96.

[16] J. Fehr, M. Fischer, B. HaAsdonk, And P. Eberhard, Greedy-based approximation of frequency-weighted Gramian matrices for model reduction in multibody dynamics, Z. Angew. Math. Mech., 93 (2013), pp. 501-519.

[17] M. Fiedler, Hankel and Loewner matrices, Linear Algebra Appl., 58 (1984), pp. 75-95.

[18] A. Frommer and V. Simoncini, Matrix functions, in Model Order Reduction: Theory, Research Aspects and Applications, W. Schilders, H. van der Vorst, and J. Rommes, eds., Math. Ind., 13 Springer, Berlin, 2008, pp. 275-303.

[19] W. Gawronski And J. JuAng, Model reduction in limited time and frequency intervals, Internat. J. Systems Sci., 21 (1990), pp. 349-376.

[20] I. Gohberg and I. Koltracht, Triangular factors of Cauchy and Vandermonde matrices, Integral Equations Operator Theroy, 26 (1996), pp. 46-59.

[21] G. Golub and C. Van Loan, Matrix Computations, 4th ed., Johns Hopkins University Press, Baltimore, MD, 2013.

[22] L. GrasedyCK, Existence of a low rank or H-matrix approximant to the solution of a Sylvester equation, Numer. Linear Algebra Appl., 11 (2004), pp. 371-389.

[23] E. J. Grimme, Krylov Projection Methods for Model Reduction, Ph.D. thesis, University of Illinois at Urbana-Champaign, 1997.

[24] S. Gugercin and A. Antoulas, A survey of model reduction by balanced truncation and some new results, Internat. J. Control, 77 (2004), pp. 748-766.

[25] S. Gugercin, A. Antoulas, and C. Beattie, $\mathcal{H}_{2}$ model reduction for large-scale dynamical systems, SIAM J. Matrix Anal. Appl., 30 (2008), pp. 609-638.

[26] S. Gugercin And J.-R. LI, Smith-type methods for balanced truncation of large systems, in Dimension Reduction of Large-Scale Systems, P. Benner, V. Mehrmann, and D. Sorensen, eds., Lect. Notes Comput. Sci. Eng. 45, Springer, Berlin, 2005, pp. 49-82.

[27] S. GütTEL, Rational Krylov approximation of matrix functions: Numerical methods and optimal pole selection, GAMM-Mitt., 36 (2013), pp. 8-31.

[28] N. Higham, Functions of Matrices: Theory and Computation, SIAM, Philadelphia, 2008.

[29] M. Hochbruck and M. Hochstenbach, Subspace Extraction for Matrix Functions, Technical report, Department of Mathematics, Case Western Reserve University, 2005.

[30] R. Horn and C. Johnson, Topics in Matrix Analysis, Cambridge, University Press, Cambridge, 1991.

[31] M. ImRAn And A. Ghafoor, Model reduction of descriptor systems using frequency limited Gramians, J. Franklin Inst., 352 (2015), pp. 33-51.

[32] I. Jaimoukha And E. Kasenally, Krylov subspace methods for solving large Lyapunov equations, SIAM J. Numer. Anal., 31 (1994), pp. 227-251.

[33] Z. JiA AND H. Lv, A posteriori error estimates of Krylov subspace approximations to matrix functions, Numer. Algorithms, 69 (2015), pp. 1-28.

[34] D. Kahaner, C. Moler, S. Nash, and G. E. Forsythe, Numerical Methods and Software, Prentice-Hall, Englewood Cliffs, NJ, 1988.

[35] L. Knizhnerman, Calculation of functions of unsymmetric matrices using Arnoldi's method, Comput. Math. Math. Phys., 31 (1992), pp. 1-9.

[36] L. Knizhnerman and V. Simoncini, A new investigation of the extended Krylov subspace method for matrix function evaluations, Numer. Linear Algebra Appl., 17 (2010), pp. 615638.

Copyright $@$ by SIAM. Unauthorized reproduction of this article is prohibited. 
[37] N. Lang, H. Mena, And J. SAak, An $L D L^{T}$ factorization based ADI algorithm for solving large scale differential matrix equations, Proc. Appl. Math. Mech., 14 (2014), pp. 827-828.

[38] N. Lang, H. Mena, And J. SaAK, On the benefits of the $L D L^{T}$ factorization for large-scale differential matrix equation solvers, Linear Algebra Appl., 480 (2015), pp. 44-71.

[39] J.-R. Li And J. White, Low Rank Solution of Lyapunov Equations, SIAM J. Matrix Anal. Appl., 24 (2002), pp. 260-280.

[40] Y. Lin And V. Simoncini, Minimal residual methods for large scale Lyapunov equations, Appl. Numer. Math., 72 (2013), pp. 52-71.

[41] B. C. Moore, Principal component analysis in linear systems: Controllability, observability, and model reduction, IEEE Trans. Automat. Control, AC-26 (1981), pp. 17-32.

[42] I. Moret And P. Novati, RD-rational approximations of the matrix exponential, BIT, 44 (2004), pp. 595-615.

[43] D. O'LeARY, The block conjugate gradient algorithm and related methods, Linear Algebra Appl., 29 (1980), pp. 293-322.

[44] T. PENZL, A cyclic low rank Smith method for large sparse Lyapunov equations, SIAM J. Sci. Comput., 21 (2000), pp. 1401-1418.

[45] T. PEnZL, Eigenvalue decay bounds for solutions of Lyapunov equations: The symmetric case, Systems Control Lett., 40 (2000), pp. 139-144.

[46] D. Petterson, A Nonlinear Optimization Approach to H2-Optimal Modeling and Control, Ph.D. thesis, Linköping University, 2013; also available from http://www.diva-portal. org/smash/get/diva2:647068/FULLTEXT01.pdf.

[47] A. RuHE, Rational Krylov sequence methods for eigenvalue computation, Linear Algebra Appl., 58 (1984), pp. 391-405.

[48] A. RuHe, The rational Krylov algorithm for nonsymmetric eigenvalue problems. III: Complex shifts for real matrices, BIT, 34 (1994), pp. 165-176.

[49] Y. SAAD, Analysis of some Krylov subspace approximations to the matrix exponential operator, SIAM J. Numer. Anal., 29 (1992), pp. 209-228.

[50] J. SAAK, Efficient Numerical Solution of Large Scale Algebraic Matrix Equations in PDE Control and Model Order Reduction, Ph.D. thesis, TU Chemnitz, 2009; also available from http://nbn-resolving.de/urn:nbn:de:bsz:ch1-200901642.

[51] D. Silvester, H. Elman, And A. Ramage, Incompressible Flow and Iterative Solver Software (IFISS), version 3.2, http://www.maths.manchester.ac.uk/ djs/ifiss/ (2012).

[52] V. Simoncini, A new iterative method for solving large-scale Lyapunov matrix equations, SIAM J. Sci. Comput., 29 (2007), pp. 1268-1288.

[53] V. Simoncini, Computational methods for linear matrix equations, SIAM Rev., to appear.

[54] T. Stykel and V. Simoncini, Krylov subspace methods for projected Lyapunov equations, Appl. Numer. Math., 62 (2012), pp. 35-50.

[55] N. Truhar and K. Veselić, Bounds on the trace of a solution to the Lyapunov equation with a general stable matrix, Systems Control Lett., 56 (2007), pp. 493-503.

[56] J. VAn Den Eshof And M. Hochbruck, Preconditioning Lanczos approximations to the matrix exponential, SIAM J. Sci. Comput., 27 (2005), pp. 1438-1457.

[57] T. Wolf And H. Panzer, The ADI iteration for Lyapunov equations implicitly performs H2 pseudo-optimal model order reduction, Internat. J. Control, 89 (2015), pp. 481-493.

[58] T. Wolf, H. Panzer, and B. Lohmann, Model order reduction by approximate balanced truncation: A unifying framework, at-Autom., 61 (2013), pp. 545-556.

Copyright (c) by SIAM. Unauthorized reproduction of this article is prohibited. 